\title{
Estimates of the reproduction number for seasonal, pandemic, and zoonotic influenza: a systematic review of the literature
}

\author{
Matthew Biggerstaff ${ }^{*}$, Simon Cauchemez ${ }^{2}$, Carrie Reed ${ }^{1}$, Manoj Gambhir ${ }^{3}$ and Lyn Finelli ${ }^{1}$
}

\begin{abstract}
Background: The potential impact of an influenza pandemic can be assessed by calculating a set of transmissibility parameters, the most important being the reproduction number (R), which is defined as the average number of secondary cases generated per typical infectious case.

Methods: We conducted a systematic review to summarize published estimates of $\mathrm{R}$ for pandemic or seasonal influenza and for novel influenza viruses (e.g. H5N1). We retained and summarized papers that estimated R for pandemic or seasonal influenza or for human infections with novel influenza viruses.

Results: The search yielded 567 papers. Ninety-one papers were retained, and an additional twenty papers were identified from the references of the retained papers. Twenty-four studies reported $51 \mathrm{R}$ values for the 1918 pandemic. The median $\mathrm{R}$ value for 1918 was 1.80 (interquartile range [IQR]: 1.47-2.27). Six studies reported seven 1957 pandemic $R$ values. The median $R$ value for 1957 was 1.65 (IQR: 1.53-1.70). Four studies reported seven 1968 pandemic $R$ values. The median $R$ value for 1968 was 1.80 (IQR: 1.56-1.85). Fifty-seven studies reported 782009 pandemic R values. The median R value for 2009 was 1.46 (IQR: 1.30-1.70) and was similar across the two waves of illness: 1.46 for the first wave and 1.48 for the second wave. Twenty-four studies reported 47 seasonal epidemic $R$ values. The median $R$ value for seasonal influenza was 1.28 (IQR: 1.19-1.37). Four studies reported six novel influenza $R$ values. Four out of six $R$ values were $<1$.

Conclusions: These $\mathrm{R}$ values represent the difference between epidemics that are controllable and cause moderate illness and those causing a significant number of illnesses and requiring intensive mitigation strategies to control. Continued monitoring of R during seasonal and novel influenza outbreaks is needed to document its variation before the next pandemic.
\end{abstract}

Keywords: Reproductive number, Influenza, Pandemics, Zoonotic influenza

\section{Background}

Annual influenza epidemics occur worldwide and cause substantial morbidity and mortality [1]. In the United States between $5 \%$ and $20 \%$ of the population are infected with influenza every year [2], resulting in between 3,000 and 49,000 influenza-associated deaths [3]. Influenza viruses are constantly changing either through the collection of minor point mutations or through major antigenic shifts. These major shifts can result in the

\footnotetext{
* Correspondence: mbiggerstaff@cdc.gov

'Epidemiology and Prevention Branch, Influenza Division, National Center for Immunization and Respiratory Diseases, Centers for Disease Control and Prevention, 1600 Clifton Road NE MS A-32, Atlanta 30333, Georgia Full list of author information is available at the end of the article
}

introduction of novel influenza viruses into the human population to which humans have little or no immunity, causing pandemics [1]. Four influenza pandemics have occurred since the beginning of the $20^{\text {th }}$ century and have ranged widely in transmissibility and clinical severity $[1,4]$.

Recognizing that the characteristics of future pandemics will be difficult to predict given the mutability of the influenza virus and the range of morbidity and mortality experienced in previous pandemics, an approach to the early assessment of influenza pandemics has been developed relying on standardized measures of transmissibility and clinical severity [5]. An important transmissibility 
parameter identified is the reproduction number (R), which is defined as the average number of secondary cases generated per typical infectious case [6,7]. $\mathrm{R}$ describes on average how many persons a case will infect, and a value of $\mathrm{R}$ greater than 1 indicates that the infection may grow or persist in the population while a value of $\mathrm{R}$ less than 1 indicates that the infection will decline in the population, although exceptions exist $[7,8]$. Many methods to calculate $\mathrm{R}$ have been described that allow for the use of epidemiologic data from different epidemic time points [7]. Some examples include estimating $R$ using the growth rate of the epidemic, the epidemic curve's size and shape, the final attack rate, or by direct observation of disease transmission from one generation to the next [7]. The population susceptibility to the infection also affects the interpretation of $R$. If $R$ is calculated in a population entirely susceptible to infection (or where an assumption about population susceptibility to infection is made), then $\mathrm{R}$ is known as the basic reproduction number $\left(\mathrm{R}_{0}\right)$. In contrast, the effective reproduction number $\left(R_{E}\right)$ is calculated in a population with underlying immunity and accounts for a population's reduced susceptibility to infection [9].

The value of $R$ characterizes the final number infected in the absence of an intervention in homogeneously mixed populations, the herd immunity threshold, and, when coupled with the generation time, defined as the interval between infections in two consecutive generations, or the serial interval, defined as the interval between the onset of symptoms in two consecutive generations), the speed with which the disease spreads in the population [10-12]. Therefore, the magnitude of $R$ plays an important role in the selection and aggressiveness of countermeasures (e.g. social distancing, treating ill individuals, or vaccination) required to slow transmission of the disease $[10,13]$.

Because $\mathrm{R}$ is used as a measure of transmissibility and informs the selection of different mitigation strategies, it is important to understand the range and uncertainty of published $R$ values. In this paper, we investigate whether published estimates of $\mathrm{R}$ differ between pandemic, seasonal, and novel influenza, we compare values of $\mathrm{R}$ calculated in differing geographic regions and settings, and we explore the assumptions and limitations of the estimation methods of $\mathrm{R}$.

\section{Methods}

We performed a literature search using the PubMed database from 1950 to January 16, 2013. The following key terms were searched: "reproduction number and influenza", "reproductive number and influenza", " $R_{0}$ and influenza", "reproduction rate and influenza", and "reproductive rate and influenza". We limited our search to articles in English. We retained articles that estimated R for pandemic or seasonal influenza or for human infections with non-human influenza viruses (e.g. H5N1). For all studies retained, we abstracted the date of publication, the year, the geographic location where the data were collected, the influenza subtype, the study population, whether it was a confined setting, the wave of the observation (if during a pandemic), the estimated value of $\mathrm{R}$, the method to identify influenza cases, and whether it was a $R_{0}$ or $R_{E}$. If multiple $R$ values were provided, we provide the median and range. Since methods to estimate the reproduction number often require a value for the generation time or the serial interval, we also report those values [14]. We classified the method used to determine influenza-associated cases into two categories: laboratory confirmed, which required the use of confirmatory testing of respiratory or blood specimens, or unconfirmed, which relied on syndromic case definitions to identify cases and required no laboratory confirmation of illnesses.

Median $R$ values and interquartile ranges (IQR) were reported for each pandemic and for the group of interpandemic seasonal epidemics. If a range of values was given for an individual study instead of a point estimate, the middle value of the range was used in the pandemic or epidemic median calculations.

\section{Results}

The search strategy initially identified 567 papers (Figure 1). Ninety-one papers were retained that estimated R for pandemic or seasonal influenza or for human infections with non-human influenza viruses (e.g. H5N1). Twenty additional papers were contributed by the references of the papers identified through the original search. In all, 111 articles were retained that presented original estimates of the reproduction number (summarized in Tables 1, 2, 3, 4, 5 and 6). Data provided in the tables are also available as .csv files in Additional files 1, 2, 3, 4, 5 and 6 .

\section{8 influenza pandemic}

The origins of the 1918 influenza A/H1N1 pandemic are unknown, and illnesses are thought to have occurred in three waves $[1,37]$. The first wave began in the Northern Hemisphere in the spring 1918 [1]. A second wave of more intense transmission occurred concurrently in North America, Europe, and Africa in fall 1918, and a third and final wave occurred in some areas of the world during winter 1919 [37,125]. The 1918 pandemic was the most deadly pandemic ever recorded, and an estimated 675,000 deaths occurred in the United States during the pandemic period. In contrast to seasonal influenza, which disproportionately affects the very young and old, those aged 20-40 years were especially affected [37]. 


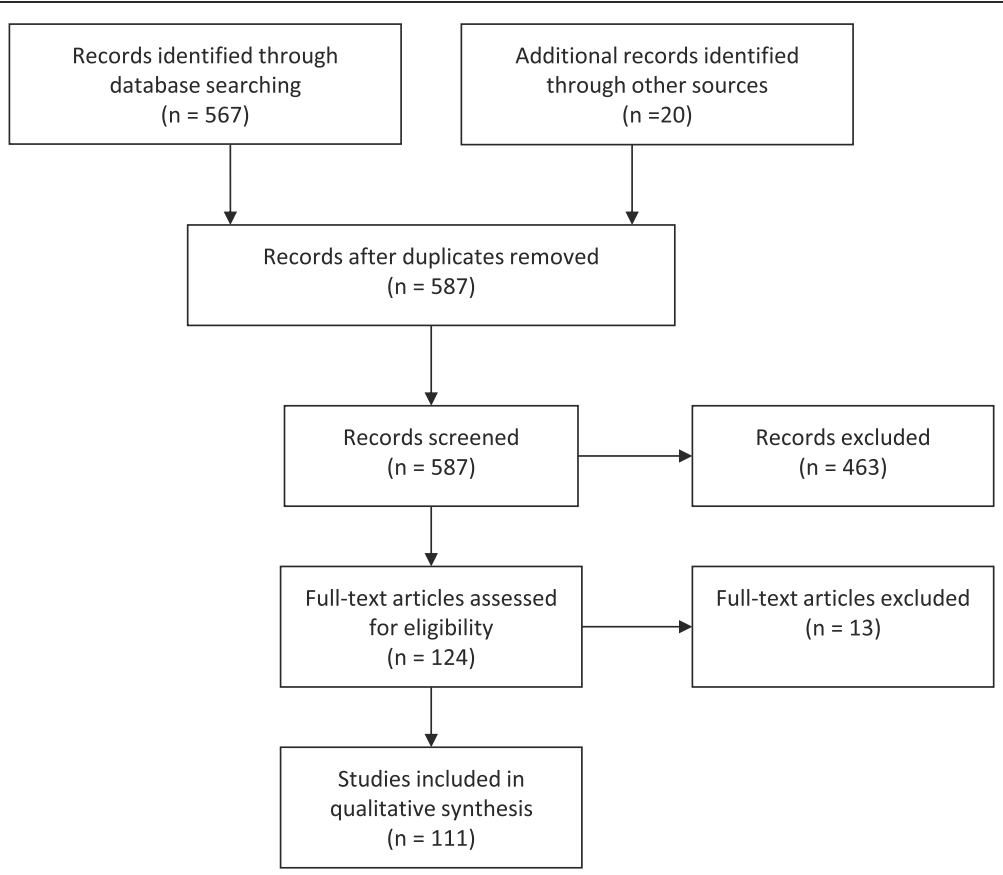

Figure 1 PRISMA flowchart of the article selection for the reproductive number and influenza literature review.

Twenty-four studies reported 51 separate 1918 pandemic values of $R$ (Table 1; Figure 2). The median point estimate of $\mathrm{R}$ in the community setting for all waves of illness was 1.80 (IQR: 1.47-2.27) (Table 1). A higher median $R$ value ( $R=3.82$; IQR: $2.68-4.84)$ was reported in confined settings, such as ships, military camps, and schools. The median values of $\mathrm{R}$ were similar between the first and subsequent waves of illness: the median value of $\mathrm{R}$ was 1.81 (IQR: $1.50-2.28$ ) for the $1^{\text {st }}$ wave, 1.73 (IQR: $1.39-2.33$ ) for the second wave, and 1.70 (IQR: 1.55-1.76) for the third wave (Table 1).

The majority of 1918 pandemic values for $\mathrm{R}$ were calculated for populations in Europe, which accounted for $58 \%$ of the $\mathrm{R}$ estimates included in this analysis. The mean generation time or serial interval used in the calculations to estimate $\mathrm{R}$ had a median value of 3.3 days, and the mean ranged from 1.5-6 days. Because the influenza virus was not discovered until 1931[1], all studies included in this review relied on reports of unconfirmed illness to identify those ill. A majority $(65 \%)$ used pneumonia-and-influenza-related hospitalizations and deaths as the case ascertainment source (Table 1).

\section{7 influenza pandemic}

The 1957 influenza A/H2N2 pandemic began in February 1957 in southern China and spread to Singapore and Hong Kong in April [1]. The virus was first isolated in the United States in June 1957 and was associated with a first wave [1,41]. The peak of the pandemic occurred during the second wave in the Northern Hemisphere in October 1957 and was followed by a third wave in January 1958. An estimated 115,000 deaths occurred in the United States during the pandemic period [37].

Six studies reported seven separate 1957 pandemic values of $\mathrm{R}$ (Table 2; Figure 3). The median point estimate of $\mathrm{R}$ in the community setting for the second wave of illnesses was 1.65 (IQR: 1.53-1.70). No $R$ values were reported for confined settings or for the $1^{\text {st }}$ or $3^{\text {rd }}$ waves of illness.

A majority (86\%) of 1957 pandemic $\mathrm{R}$ values were calculated for populations in Europe. The mean generation time or serial interval used in the calculations to determine $\mathrm{R}$ had a median value of 3.5 days, and the mean ranged from 2.6-4.1 days. All studies but one included in this review relied on an unconfirmed illnesses to identify those ill. The other study relied on the final attack rate as determined by serological methods (Table 2).

\section{8 influenza pandemic}

The 1968 influenza A/H3N2 pandemic began in Hong Kong in July 1968. Large single waves were reported in the Northern Hemisphere between September 1968 and April 1969 (with peaks occurring in December and January) and in the Southern Hemisphere between June and September 1969. Some countries in the Northern Hemisphere, such as the United Kingdom, did not have an 
Table 1 Reproduction numbers from the 1918 Influenza A/H1N1 Pandemic

\begin{tabular}{|c|c|c|c|c|c|c|c|c|c|}
\hline Location & Wave $^{a}$ & $\begin{array}{c}\text { Study } \\
\text { population }\end{array}$ & $\begin{array}{l}\text { Mean } \\
\text { GT/SI }^{b}\end{array}$ & $\begin{array}{c}\text { Reproduction } \\
\text { Number (R) }\end{array}$ & $95 \% \mathrm{Cl}^{\mathrm{c}}$ & $\begin{array}{l}\text { Basic or } \\
\text { effective }\end{array}$ & Case definition & Reference & $\begin{array}{c}\text { Year } \\
\text { published }\end{array}$ \\
\hline Australia & $1 s t$ & Community & 2.6 & 1.80 & $1.6-2.0$ & Basic & $\begin{array}{c}\text { Unconfirmed } \\
\text { hospitalizations/deaths }\end{array}$ & [15] & 2008 \\
\hline Brazil & $1 s t$ & Community & 4 & 2.68 & & Basic & Unconfirmed illness & [16] & 2007 \\
\hline Canada & $1 s t$ & Community & 3 & 1.50 & $1.5-1.5$ & Basic & Unconfirmed deaths & {$[17]$} & 2011 \\
\hline Canada & $1 s t$ & Community & 6 & 2.1 & $2.1-2.1$ & Basic & Unconfirmed deaths & {$[17]$} & 2011 \\
\hline Colombia & $1 s t$ & Community & 3 & $1.4-1.5$ & & Effective & Unconfirmed deaths & [18] & 2012 \\
\hline Colombia & $1 s t$ & Community & 4 & $1.5-1.7$ & & Effective & Unconfirmed deaths & [18] & 2012 \\
\hline Denmark & $1 s t$ & Community & 2.6 & $2.2-2.4$ & & Effective & Unconfirmed illness & [19] & 2008 \\
\hline Denmark & $1 s t$ & Community & 4 & $2.8-3.0$ & & Effective & Unconfirmed illness & [19] & 2008 \\
\hline Denmark & $1 s t$ & Community & 2.6 & $2.8-4.0$ & & Effective & $\begin{array}{l}\text { Unconfirmed } \\
\text { hospitalizations }\end{array}$ & [19] & 2008 \\
\hline Denmark & $1 s t$ & Community & 4 & $3.6-5.4$ & & Effective & $\begin{array}{l}\text { Unconfirmed } \\
\text { hospitalizations }\end{array}$ & [19] & 2008 \\
\hline Italy & $1 s t$ & Community & 3 & 1.03 & $1.00-1.08$ & Basic & $\begin{array}{l}\text { Unconfirmed } \\
\text { hospitalizations }\end{array}$ & {$[20]$} & 2011 \\
\hline Mexico & $1 s t$ & Community & 3 & 1.30 & & Effective & Unconfirmed deaths & [21] & 2010 \\
\hline Peru & $1 s t$ & Community & 3 & 1.38 & $1.37-1.40$ & Effective & Unconfirmed deaths & [22] & 2011 \\
\hline Switzerland & $1 s t$ & Community & 3.11 & 1.49 & $1.45-1.53$ & Basic & $\begin{array}{l}\text { Unconfirmed } \\
\text { hospitalizations }\end{array}$ & [23] & 2006 \\
\hline Switzerland & $1 s t$ & Community & 3.4 & 1.50 & & Basic & Unconfirmed deaths & [24] & 2009 \\
\hline $\begin{array}{l}\text { United } \\
\text { Kingdom }\end{array}$ & $1 s t$ & Community & 2.6 & 1.7 & & Basic & Unconfirmed deaths & [10] & 2006 \\
\hline $\begin{array}{l}\text { United } \\
\text { Kingdom }\end{array}$ & $1 s t$ & Community & 4.1 & 2.10 & & Effective & Unconfirmed illness & [25] & 2006 \\
\hline $\begin{array}{l}\text { United } \\
\text { Kingdom }\end{array}$ & $1 s t$ & Community & 6 & 2.00 & & Basic & Unconfirmed illness & {$[26]$} & 2005 \\
\hline $\begin{array}{l}\text { United } \\
\text { Kingdom }\end{array}$ & $1 s t$ & Community & NR & $1.16-2.94$ & & Effective & Unconfirmed illness & [27] & 2010 \\
\hline $\begin{array}{l}\text { United } \\
\text { Kingdom }\end{array}$ & $1 \mathrm{st}$ & Students & NR & $1.43-5.36$ & & Effective & Unconfirmed illness & [27] & 2010 \\
\hline USA & $1 s t$ & Community & 4 & $1.34-3.21$ & & Effective & Unconfirmed illness & {$[28]$} & 2008 \\
\hline Various & $1 \mathrm{st}$ & Community & 4 & $1.2-3.0$ & & Effective & Unconfirmed illness & [29] & 2007 \\
\hline \multirow[t]{2}{*}{ Various } & $1 s t$ & Community & 4 & $2.1-7.5$ & & Effective & Unconfirmed illness & [29] & 2007 \\
\hline & $1 s t$ & Sailors & 4 & 4.97 & & Effective & Unconfirmed illness & {$[28]$} & 2008 \\
\hline Canada & 2nd & Community & 3.6 & 2.26 & $1.95-2.63$ & Basic & Unconfirmed illness & {$[30]$} & 2010 \\
\hline Canada & 2nd & Community & 3.6 & 1.49 & $1.42-1.55$ & Basic & Unconfirmed illness & {$[30]$} & 2010 \\
\hline Canada & 2nd & Community & 3 & 2.40 & $2.4-2.5$ & Basic & Unconfirmed deaths & {$[17]$} & 2011 \\
\hline Canada & 2nd & Community & 6 & 4.3 & $4.2-4.4$ & Basic & Unconfirmed deaths & [17] & 2011 \\
\hline Denmark & 2nd & Community & 2.6 & $1.22-1.24$ & & Effective & Unconfirmed illness & [19] & 2008 \\
\hline Denmark & 2nd & Community & 4 & $1.29-1.33$ & & Effective & Unconfirmed illness & [19] & 2008 \\
\hline Denmark & 2nd & Community & 2.6 & $1.2-1.3$ & & Effective & $\begin{array}{l}\text { Unconfirmed } \\
\text { hospitalizations }\end{array}$ & [19] & 2008 \\
\hline Denmark & 2nd & Community & 4 & $1.3-1.4$ & & Effective & $\begin{array}{l}\text { Unconfirmed } \\
\text { hospitalizations }\end{array}$ & [19] & 2008 \\
\hline Germany & 2nd & Community & 1 & 1.58 & $0.03-10.3$ & Basic & Unconfirmed deaths & [31] & 2007 \\
\hline Germany & 2nd & Community & 3 & 2.52 & $0.75-5.85$ & Basic & Unconfirmed deaths & {$[31]$} & 2007 \\
\hline Germany & $2 n d$ & Community & 5 & 3.41 & $1.91-5.57$ & Basic & Unconfirmed deaths & {$[31]$} & 2007 \\
\hline
\end{tabular}


Table 1 Reproduction numbers from the 1918 Influenza A/H1N1 Pandemic (Continued)

\begin{tabular}{|c|c|c|c|c|c|c|c|c|c|}
\hline Italy & 2nd & Community & 3 & 1.38 & $1.3-1.5$ & Basic & $\begin{array}{c}\text { Unconfirmed } \\
\text { hospitalizations }\end{array}$ & [20] & 2011 \\
\hline Mexico & 2nd & Community & 3 & 1.30 & & Effective & Unconfirmed deaths & [21] & 2010 \\
\hline $\begin{array}{l}\text { New } \\
\text { Zealand }\end{array}$ & 2nd & Military & $>1.5$ & $1.3-3.1$ & & Basic & $\begin{array}{l}\text { Unconfirmed } \\
\text { hospitalizations }\end{array}$ & [32] & 2006 \\
\hline Switzerland & 2nd & Community & 2.28 & 3.75 & $3.6-3.9$ & Effective & $\begin{array}{c}\text { Unconfirmed } \\
\text { hospitalizations }\end{array}$ & [23] & 2006 \\
\hline Switzerland & 2nd & Community & 3.4 & 2.40 & & Basic & Unconfirmed deaths & [24] & 2009 \\
\hline $\begin{array}{l}\text { United } \\
\text { Kingdom }\end{array}$ & 2nd & Community & 3 & 1.39 & $1.36-1.43$ & Effective & Unconfirmed deaths & [33] & 2008 \\
\hline $\begin{array}{c}\text { United } \\
\text { Kingdom }\end{array}$ & 2nd & Community & 6 & 1.84 & $1.75-1.92$ & Effective & Unconfirmed deaths & [33] & 2008 \\
\hline $\begin{array}{l}\text { United } \\
\text { Kingdom }\end{array}$ & 2nd & Community & 6 & 1.55 & & Basic & Unconfirmed illness & [26] & 2005 \\
\hline $\begin{array}{l}\text { United } \\
\text { Kingdom }\end{array}$ & 2nd & Community & 2.6 & 1.50 & & Basic & Unconfirmed deaths & {$[10]$} & 2006 \\
\hline USA & 2nd & Community & 2.5 & 2.14 & & Basic & Unconfirmed deaths & [34] & 2009 \\
\hline USA & 2nd & Community & NR & 2.20 & $1.55-2.84$ & Effective & Unconfirmed illness & [35] & 2007 \\
\hline USA & 2nd & Community & 4 & 2.00 & $1.7-2.3$ & Effective & Unconfirmed deaths & [36] & 2004 \\
\hline USA & 2nd & Community & 2.85 & 1.73 & & Effective & Unconfirmed deaths & [14] & 2007 \\
\hline $\begin{array}{c}\text { United } \\
\text { Kingdom }\end{array}$ & $3 r d$ & Community & 3 & 1.39 & $1.29-1.49$ & Effective & Unconfirmed deaths & [33] & 2008 \\
\hline $\begin{array}{c}\text { United } \\
\text { Kingdom }\end{array}$ & $3 r d$ & Community & 6 & 1.82 & $1.61-2.05$ & Effective & Unconfirmed deaths & [33] & 2008 \\
\hline $\begin{array}{l}\text { United } \\
\text { Kingdom }\end{array}$ & $3 r d$ & Community & 6 & 1.70 & & Basic & Unconfirmed illness & [26] & 2005 \\
\hline
\end{tabular}

${ }^{a}$ The first wave of illnesses began in the Northern Hemisphere in the spring 1918 [1]. A second wave of more intense transmission occurred concurrently in North America, Europe, and Africa in the Fall of 1918 while a third and final wave of activity occurred in some areas of the world during the winter of 1919 [37].

${ }^{\mathrm{b}}$ The generation time (GT) or serial interval (SI) assumed in the reproduction number estimation.

${ }^{\mathrm{C} C o n f i d e n c e ~ i n t e r v a l . ~}$

$\mathrm{NR}=$ Not reported.

This table is also available as a .csv file as Additional file 1.

outbreak of H3N2 until the winter of 1969-70. In all, an estimated 110,000 deaths occurred in the United States during the pandemic period [37].

Four studies reported seven separate 1968 pandemic values of $\mathrm{R}$ (Table 3; Figure 3). The median point estimate of $\mathrm{R}$ in the community setting for all waves of illness was 1.80 (IQR: 1.56-1.85) (Table 3). Only two values for $\mathrm{R}$ in confined settings were reported, and the median value was 1.39 . Two values of $\mathrm{R}$ were reported in a community setting during the first wave and three during the second wave. The median value of $\mathrm{R}$ during the $1^{\text {st }}$ wave was 1.56 and 1.68 during the $2^{\text {nd }}$ wave (Table 3).

The 1968 pandemic values for $\mathrm{R}$ were calculated among populations in diverse geographic locations, mainly because of one study that calculated separate values for over 25 locations, such as Africa, Asia, and South America (the overall estimate for $\mathrm{R}$ is included in
Table 3) [43]. The mean generation time or serial interval used in the calculations to determine $\mathrm{R}$ had a median value of 4 days with little variation. The studies for the 1968 pandemic included in this review relied on a mix of laboratory-confirmed, unconfirmed illnesses, or serologically-confirmed infections to identify those ill (Table 3).

\section{The 2009 influenza pandemic}

The 2009 influenza A/H1N1 pandemic began in Mexico in the late winter or early spring 2009 [44]. The United States and the United Kingdom experienced a first wave of illnesses in the spring followed by a second wave during the fall [4]. However, a number of other countries, especially in the Southern Hemisphere, only experienced a single wave of illnesses [100]. In all, an estimated 12,000 deaths occurred in the United States during the first year of pandemic circulation [126]. 
Table 2 Reproduction numbers from the 1957 influenza A/H2N2 pandemic

\begin{tabular}{|c|c|c|c|c|c|c|c|c|c|}
\hline Location & Wave $^{a}$ & $\begin{array}{c}\text { Study } \\
\text { population }\end{array}$ & $\begin{array}{l}\text { Mean } \\
\mathrm{GT} / \mathrm{SI}^{\mathrm{b}}\end{array}$ & $\begin{array}{l}\text { Reproduction } \\
\text { number (R) }\end{array}$ & $95 \% \mathrm{Cl}^{\mathrm{c}}$ & $\begin{array}{l}\text { Basic or } \\
\text { effective }\end{array}$ & Case definition & Reference & $\begin{array}{c}\text { Year } \\
\text { published }\end{array}$ \\
\hline Netherlands & 2nd & Community & 3 & 1.39 & & Basic & Unconfirmed deaths & [38] & 2010 \\
\hline $\begin{array}{l}\text { United } \\
\text { Kingdom }\end{array}$ & 2nd & Community & 2.6 & 1.70 & & Basic & Unconfirmed deaths & [10] & 2006 \\
\hline $\begin{array}{l}\text { United } \\
\text { Kingdom }\end{array}$ & 2nd & Community & 3 & $1.5-1.6$ & & Basic & Unconfirmed illness & [39] & 2008 \\
\hline $\begin{array}{l}\text { United } \\
\text { Kingdom }\end{array}$ & 2nd & Community & 4 & $1.7-1.8$ & & Basic & Unconfirmed illness & [39] & 2008 \\
\hline $\begin{array}{l}\text { United } \\
\text { Kingdom }\end{array}$ & 2nd & Community & 4.1 & 1.50 & & Effective & Unconfirmed illness & [25] & 2006 \\
\hline $\begin{array}{l}\text { United } \\
\text { Kingdom }\end{array}$ & 2nd & Community & NR & 1.65 & & Basic & $\begin{array}{l}\text { Serology confirmed } \\
\text { infection }\end{array}$ & [26] & 2005 \\
\hline USA & 2nd & Community & 4 & 1.70 & & Basic & Unconfirmed illness & [40] & 2004 \\
\hline
\end{tabular}

Median reproduction number for the 1957 pandemic: 1.65; Interquartile range 1.53-1.70

${ }^{a}$ The 1957 influenza A/H2N2 pandemic began in February 1957 in southern China and spread to Singapore and Hong Kong in April [1]. The virus was first isolated in the United States in June 1957 and was associated with a mild first wave of illnesses [1,41]. The peak of the pandemic occurred during the second wave in the Northern Hemisphere in October 1957 and was followed by a third wave in January 1958.

${ }^{\mathrm{b}}$ The generation time (GT) or serial interval (SI) assumed in the reproduction number estimation.

${ }^{\mathrm{C}}$ Confidence interval.

NR $=$ Not reported

This table is also available as a .csv file as Additional file 2.

Fifty-seven studies reported 78 separate 2009 pandemic values of $R$ (Table 4; Figure 4). The median point estimate of $\mathrm{R}$ in the community setting for all waves of illness was 1.46 (IQR: 1.30-1.70) while a higher median $R$ value $(R=1.96$; IQR: $1.50-2.23)$ was reported in confined settings, such as military or summer camps, schools, and night clubs. The value of $\mathrm{R}$ was similar across the two distinct waves of illness: the median value of $\mathrm{R}$ was 1.47 (IQR: 1.31-1.71) for the first wave and 1.48 (IQR: 1.30-1.66) for the second wave (Table 4).

A majority of 2009 pandemic values for $\mathrm{R}$ were calculated for populations in North America (30\%) and Asia

Table 3 Reproduction numbers from the 1968 influenza A/H3N2 pandemic

\begin{tabular}{|c|c|c|c|c|c|c|c|c|c|}
\hline Location & Wave $^{a}$ & $\begin{array}{c}\text { Study } \\
\text { population }\end{array}$ & $\begin{array}{l}\text { Mean } \\
\text { GT/SI }\end{array}$ & $\begin{array}{l}\text { Reproduction } \\
\text { number (R) }\end{array}$ & $95 \% \mathrm{Cl}^{\mathrm{c}}$ & $\begin{array}{l}\text { Basic or } \\
\text { effective }\end{array}$ & Case definition & Reference & $\begin{array}{c}\text { Year } \\
\text { published }\end{array}$ \\
\hline $\begin{array}{l}\text { Hong } \\
\text { Kong }\end{array}$ & $1 \mathrm{st}$ & Community & 2.95 & 1.89 & & Basic & Unconfirmed illness & {$[42]$} & 1986 \\
\hline various & $1 \mathrm{st}$ & Community & 4 & $1.06-2.06$ & & Basic & $\begin{array}{l}\text { Serology; laboratory confirmed } \\
\text { illness; unconfirmed illness }\end{array}$ & {$[43]$} & 2010 \\
\hline various & $1 \mathrm{st}$ & $\begin{array}{l}\text { Confined } \\
\text { settings }\end{array}$ & 4 & $1.08-1.62$ & & Basic & $\begin{array}{l}\text { Serology; laboratory confirmed } \\
\text { illness; unconfirmed illness }\end{array}$ & {$[43]$} & 2010 \\
\hline $\begin{array}{l}\text { United } \\
\text { Kingdom }\end{array}$ & $1 \mathrm{st}$ & Community & 4.1 & 1.80 & & Effective & Unconfirmed illness & {$[25]$} & 2006 \\
\hline $\begin{array}{l}\text { United } \\
\text { Kingdom }\end{array}$ & 2nd & Community & $N R$ & 1.85 & & Effective & Serology confirmed infection & {$[26]$} & 2005 \\
\hline various & 2nd & Community & 4 & $1.08-2.02$ & & Effective & $\begin{array}{l}\text { Serology; laboratory confirmed } \\
\text { illness; unconfirmed illness }\end{array}$ & [43] & 2010 \\
\hline various & $2 n d$ & $\begin{array}{l}\text { Confined } \\
\text { settings }\end{array}$ & 4 & 1.43 & $1.23-1.63$ & Effective & $\begin{array}{l}\text { Serology; laboratory confirmed } \\
\text { illness; unconfirmed illness }\end{array}$ & [43] & 2010 \\
\hline
\end{tabular}

Median reproduction number for the 1968 pandemic: 1.80; Interquartile range 1.56-1.85.

${ }^{a}$ The 1968 influenza A/H3N2 pandemic began in Hong Kong in July 1968. Large single waves of illness were reported in the Northern Hemisphere between September 1968 and April 1969 (with peaks occurring in December 1968-January 1969). Large single waves of illnesses were reported in the Southern Hemisphere between June and September 1969. Some countries in the Northern Hemisphere, such as the United Kingdom, did not have an outbreak of H3N2 until the winter of 1969-70.

${ }^{\mathrm{b}}$ The generation time (GT) or serial interval (SI) assumed in the reproduction number estimation.

${ }^{\mathrm{c} C o n f i d e n c e ~ i n t e r v a l . ~}$

$\mathrm{NR}=$ Not reported.

This table is also available as a .csv file as Additional file 3. 
Table 4 Reproduction numbers from the 2009 influenza A/H1N1 pandemic

\begin{tabular}{|c|c|c|c|c|c|c|c|c|c|}
\hline Location & Wave $^{a}$ & $\begin{array}{c}\text { Study } \\
\text { population }\end{array}$ & $\begin{array}{l}\text { Mean } \\
\mathrm{GT}_{\mathrm{G}} \mathrm{SI}^{\mathrm{b}}\end{array}$ & $\begin{array}{c}\text { Reproduction } \\
\text { number (R) }\end{array}$ & $95 \% \mathrm{Cl}^{\mathrm{C}}$ & $\begin{array}{l}\text { Basic or } \\
\text { effective }\end{array}$ & Case definition & Reference & $\begin{array}{c}\text { Year } \\
\text { published }\end{array}$ \\
\hline Mexico & 0 & Community & 1.91 & 1.25 & $0.76-1.74$ & Basic & Laboratory confirmed illness & {$[44]$} & 2011 \\
\hline Australia & $1 s t$ & Community & 2.8 & 1.50 & $1.50-2.70$ & Effective & Laboratory confirmed illness & {$[45]$} & 2010 \\
\hline Australia & $1 s t$ & Community & 2.8 & 1.20 & $1.0-1.4$ & Effective & Laboratory confirmed illness & {$[45]$} & 2010 \\
\hline Australia & $1 s t$ & Community & 2.9 & 2.40 & $2.3-2.4$ & Effective & Laboratory confirmed illness & [46] & 2009 \\
\hline Australia, rural & $1 s t$ & Community & 2.9 & 1.28 & $1.26-1.30$ & Effective & Laboratory confirmed illness & {$[47]$} & 2011 \\
\hline Australia, urban & $1 s t$ & Community & 2.9 & 1.26 & $1.22-1.30$ & Effective & Laboratory confirmed illness & {$[47]$} & 2011 \\
\hline Canada & $1 s t$ & Community & 1.91 & 1.30 & $1.12-1.47$ & Basic & Laboratory confirmed illness & [48] & 2010 \\
\hline Canada & $1 s t$ & Community & 2.78 & 2.21 & $1.98-2.50$ & Basic & Laboratory confirmed illness & [49] & 2012 \\
\hline Canada & $1 s t$ & Community & 3.6 & 1.63 & $1.31-1.96$ & Basic & Laboratory confirmed illness & [48] & 2010 \\
\hline Canada & $1 s t$ & Community & 4.31 & 1.31 & $1.25-1.38$ & Basic & Laboratory confirmed illness & {$[50]$} & 2010 \\
\hline Chile & $1 s t$ & Community & 2.5 & 1.80 & $1.6-2.0$ & Effective & Unconfirmed emergency room visits & {$[51]$} & 2010 \\
\hline Chile, central & $1 s t$ & Community & 3 & 1.32 & $1.27-1.37$ & Effective & Unconfirmed hospitalizations & {$[52]$} & 2012 \\
\hline Chile, northern & $1 s t$ & Community & 3 & 1.19 & $1.13-1.24$ & Effective & Unconfirmed hospitalizations & [52] & 2012 \\
\hline Chile, southern & $1 s t$ & Community & 3 & 1.58 & $1.45-1.72$ & Effective & Unconfirmed hospitalizations & {$[52]$} & 2012 \\
\hline China & $1 s t$ & Community & 2.6 & 1.25 & $1.22-1.28$ & Effective & Laboratory confirmed illness & [53] & 2012 \\
\hline China & $1 s t$ & Community & 4.31 & 1.53 & $1.45-1.60$ & Basic & Laboratory confirmed illness & {$[54]$} & 2012 \\
\hline China & $1 s t$ & Community & NR & 1.68 & & Basic & Laboratory confirmed illness & {$[55]$} & 2011 \\
\hline Hong Kong & $1 s t$ & Community & 3 & 1.70 & $1.6-1.8$ & Effective & Laboratory confirmed illness & {$[56]$} & 2010 \\
\hline Hong Kong & $1 s t$ & Community & 3.2 & 1.45 & $1.4-1.5$ & Effective & Laboratory confirmed illness & {$[57]$} & 2010 \\
\hline Israel & $1 s t$ & Community & 2.92 & 1.06 & $0.97-1.16$ & Effective & Laboratory confirmed illness & [58] & 2011 \\
\hline Italy & $1 s t$ & Community & 2.6 & 1.30 & $1.23-1.32$ & Effective & Unconfirmed illness & {$[59]$} & 2012 \\
\hline Japan & $1 s t$ & School & 1.9 & 2.30 & $2.0-2.6$ & Effective & Laboratory confirmed illness & {$[60]$} & 2009 \\
\hline Japan & $1 s t$ & Community & 2.7 & 1.28 & $1.23-1.33$ & Effective & Laboratory confirmed illness & {$[60]$} & 2009 \\
\hline Mexico & $1 s t$ & Community & 1.91 & 1.58 & $1.34-2.04$ & Basic & Unconfirmed illness & {$[61]$} & 2009 \\
\hline Mexico & $1 s t$ & Community & 1.96 & 1.42 & & Basic & Unconfirmed illness & {$[62]$} & 2010 \\
\hline Mexico & $1 s t$ & Community & 2.6 & 1.40 & $1.2-1.9$ & Basic & Laboratory confirmed illness & {$[61]$} & 2009 \\
\hline Mexico & $1 s t$ & Community & 2.6 & 1.22 & $1.05-1.60$ & Basic & Laboratory confirmed illness & {$[61]$} & 2009 \\
\hline Mexico & $1 s t$ & Community & 3 & 1.80 & $1.78-1.81$ & Effective & Unconfirmed illness & {$[63]$} & 2011 \\
\hline Mexico & $1 s t$ & Community & 3 & 1.43 & $1.29-1.57$ & Effective & Laboratory confirmed illness & [64] & 2009 \\
\hline Mexico & $1 s t$ & Community & 3.1 & 2.20 & $2.1-2.4$ & Effective & Laboratory confirmed illness & {$[65]$} & 2009 \\
\hline Mexico & $1 s t$ & Community & 3.5 & 2.30 & $2.1-2.5$ & Basic & Laboratory confirmed illness & [11] & 2009 \\
\hline Mexico & $1 s t$ & Community & 3.6 & 1.75 & $1.6-1.9$ & Basic & Seeding from Mexico & {$[66]$} & 2009 \\
\hline Mexico & $1 s t$ & Community & 4.1 & 3.10 & $2.9-3.5$ & Effective & Laboratory confirmed illness & {$[65]$} & 2009 \\
\hline Mexico City & $1 s t$ & Community & 3 & 1.72 & & Basic & Laboratory confirmed illness & {$[67]$} & 2009 \\
\hline Morocco & $1 s t$ & Community & 2.3 & 1.44 & $1.32-1.56$ & Basic & Laboratory confirmed illness & {$[68]$} & 2012 \\
\hline Morocco & $1 s t$ & Community & 2.7 & 1.40 & $1.34-1.48$ & Basic & Laboratory confirmed illness & {$[68]$} & 2012 \\
\hline Netherlands & $1 s t$ & Community & 3 & 0.50 & & Effective & Laboratory confirmed illness & {$[69]$} & 2009 \\
\hline New Zealand & 1st & Community & 2.7 & 1.25 & $1.07-1.47$ & Effective & Laboratory confirmed illness & [70] & 2011 \\
\hline New Zealand & $1 s t$ & Community & 2.8 & 1.96 & $1.80-2.15$ & Effective & Laboratory confirmed illness & [71] & 2009 \\
\hline New Zealand & $1 s t$ & Community & 2.8 & 1.55 & $1.16-1.86$ & Effective & $\begin{array}{l}\text { Laboratory confirmed illness; } \\
\text { unconfirmed illness }\end{array}$ & [72] & 2010 \\
\hline North America & $1 s t$ & Community & 2.7 & $1.3-2.1$ & & Basic & Laboratory confirmed illness & [73] & 2010 \\
\hline Peru & $1 s t$ & Community & 2.8 & 1.37 & $1.33-1.41$ & Effective & Laboratory confirmed illness & [74] & 2009 \\
\hline Peru & $1 s t$ & Community & 3 & 1.30 & $1.3-1.3$ & Effective & Unconfirmed illness & {$[75]$} & 2011 \\
\hline
\end{tabular}


Table 4 Reproduction numbers from the 2009 influenza A/H1N1 pandemic (Continued)

\begin{tabular}{|c|c|c|c|c|c|c|c|c|c|}
\hline Peru, Lima & 1 st & Community & 3 & 1.70 & $1.6-1.7$ & Effective & Unconfirmed illness & [75] & 2011 \\
\hline Singapore & $1^{\text {st }}$ & Dance club & 1.91 & $1.9-2.1$ & & Basic & Laboratory confirmed illness & [76] & 2010 \\
\hline Singapore & $1 \mathrm{st}$ & Military & NR & 1.91 & $1.50-2.36$ & Effective & $\begin{array}{l}\text { Laboratory confirmed and } \\
\text { unconfirmed illness }\end{array}$ & [77] & 2010 \\
\hline South Africa & $1 \mathrm{st}$ & Community & 2.3 & 1.43 & $1.38-1.49$ & Effective & Laboratory confirmed illness & [78] & 2012 \\
\hline South Africa & $1 s t$ & Community & 2.78 & 1.47 & $1.30-1.72$ & Effective & Laboratory confirmed illness & [78] & 2012 \\
\hline South Africa & 1 st & Community & 2.78 & 1.42 & $1.20-1.71$ & Effective & Laboratory confirmed illness & [78] & 2012 \\
\hline $\begin{array}{l}\text { Southern } \\
\text { Hemisphere }\end{array}$ & $1 s t$ & Community & 1.9 & $1.16-1.53$ & & Effective & Laboratory confirmed illness & [79] & 2010 \\
\hline $\begin{array}{l}\text { Southern } \\
\text { Hemisphere }\end{array}$ & $1 s t$ & Community & 2.60 & 1.33 & $1.28-1.45$ & Basic & $\begin{array}{l}\text { Laboratory confirmed and } \\
\text { unconfirmed illness }\end{array}$ & [80] & 2011 \\
\hline Taiwan & 1 st & Community & 1.91 & 1.14 & $1.04-1.25$ & Effective & Laboratory confirmed illness & [81] & 2011 \\
\hline Taiwan & $1 \mathrm{st}$ & Community & NR & 1.16 & $0.98-1.34$ & Effective & Serology confirmed infection & [82] & 2011 \\
\hline Thailand & $1 \mathrm{st}$ & Community & 1.9 & 1.78 & $1.67-1.89$ & Basic & Laboratory confirmed illness & [83] & 2009 \\
\hline Thailand & 1 st & Community & 2.6 & 2.07 & $1.92-2.22$ & Basic & Laboratory confirmed illness & [83] & 2009 \\
\hline $\begin{array}{l}\text { United } \\
\text { Kingdom }\end{array}$ & $1 \mathrm{st}$ & School & 2.2 & 1.33 & $1.11-1.56$ & Effective & Laboratory confirmed illness & [84] & 2012 \\
\hline $\begin{array}{l}\text { United } \\
\text { Kingdom }\end{array}$ & $1 \mathrm{st}$ & Community & 2.5 & 1.44 & $1.27-1.63$ & Effective & Laboratory confirmed illness & [85] & 2009 \\
\hline USA & $1 \mathrm{st}$ & Community & 2.2 & 1.70 & $1.4-2.1$ & Basic & Laboratory confirmed illness & [86] & 2009 \\
\hline USA & $1 \mathrm{st}$ & Community & 2.6 & 2.20 & $1.4-2.5$ & Basic & Laboratory confirmed illness & [86] & 2009 \\
\hline USA & $1 \mathrm{st}$ & School & 2.7 & 3.30 & $3.0-3.6$ & Effective & Unconfirmed illness & [87] & 2009 \\
\hline USA & $1 s t$ & Community & 3.5 & $1.3-2.0$ & $1.0-2.2$ & Basic & Laboratory confirmed illness & [11] & 2009 \\
\hline USA & $1 \mathrm{st}$ & $\begin{array}{l}\text { Camp } \\
\text { attendees }\end{array}$ & 7 & 2.20 & $1.4-3.3$ & Effective & Unconfirmed illness & [88] & 2011 \\
\hline Vietnam & $1 \mathrm{st}$ & Community & 1.9 & 1.50 & $1.5-1.6$ & Basic & Laboratory confirmed illness & [89] & 2010 \\
\hline Vietnam & $1 \mathrm{st}$ & Community & 3.6 & 2.00 & $1.9-2.2$ & Basic & Laboratory confirmed illness & [89] & 2010 \\
\hline worldwide & $1 \mathrm{st}$ & Community & 2.67 & $1-2$ & & Effective & Laboratory confirmed illness & [90] & 2011 \\
\hline China & 2nd & Community & 4 & 1.66 & $1.27-2.05$ & Effective & confirmed hospitalizations & [91] & 2012 \\
\hline China & 2nd & Community & 4.3 & 1.70 & $1.4-1.9$ & Effective & Laboratory confirmed illness & [92] & 2010 \\
\hline France & 2nd & Military & 2.9 & $1.5-1.6$ & & Effective & Unconfirmed illness & [93] & 2012 \\
\hline Iran & 2nd & school & NR & 1.28 & $1.05-1.54$ & Basic & Unconfirmed illness & [94] & 2012 \\
\hline Italy & 2nd & Community & 2.5 & 1.33 & & Effective & Unconfirmed illness & [95] & 2011 \\
\hline Japan & 2nd & Community & 3 & 1.48 & $1.41-1.56$ & Effective & Unconfirmed illness & [96] & 2012 \\
\hline Mexico & 2nd & Community & 3 & 1.62 & $1.61-1.63$ & Effective & Unconfirmed illness & [63] & 2011 \\
\hline Reunion Island & 2nd & Community & 2.8 & 1.26 & $1.08-1.49$ & Effective & Unconfirmed illness & [97] & 2010 \\
\hline Taiwan & 2nd & Community & 1.91 & 1.02 & $1.01-1.02$ & Effective & Laboratory confirmed illness & [81] & 2011 \\
\hline Taiwan & 2nd & Community & $N R$ & 1.87 & $1.68-2.06$ & Effective & Serology confirmed infection & [82] & 2011 \\
\hline $\begin{array}{l}\text { United } \\
\text { Kingdom }\end{array}$ & 2nd & Community & 2.5 & 1.30 & $1.2-1.5$ & Effective & Laboratory confirmed illness & [98] & 2010 \\
\hline Mexico & $3 r d$ & Community & 3 & 1.24 & $1.23-1.24$ & Effective & Unconfirmed illness & [63] & 2011 \\
\hline various & & Community & NR & 1.30 & $1.1-1.4$ & Effective & Serology confirmed infection & [99] & 2012 \\
\hline
\end{tabular}

Median reproduction number for the 2009 pandemic: 1.46; Interquartile range 1.30-1.70

${ }^{a}$ The 2009 influenza A/H1N1 pandemic began in Mexico in the late winter or early spring of 2009 [44]. The United States and the United Kingdom experienced a first wave of illnesses in the Spring of 2009 followed by a second wave during the Fall of 2009 [4]. However, unlike these three countries, a number of countries, especially in the Southern Hemisphere, only experienced a single wave of illnesses [100].

${ }^{b}$ The generation time (GT) or serial interval (SI) assumed in the reproduction number estimation.

${ }^{\mathrm{C}}$ Confidence interval.

$\mathrm{NR}=$ Not reported

This table is also available as a .csv file as Additional file 4. 
Table 5 Reproduction numbers from seasonal influenza epidemics

\begin{tabular}{|c|c|c|c|c|c|c|c|c|c|}
\hline Year(s) & $\begin{array}{l}\text { Type/ } \\
\text { Subtype }\end{array}$ & $\begin{array}{l}\text { Study } \\
\text { population }\end{array}$ & $\begin{array}{l}\text { Mean } \\
\mathrm{GT} /\left.\mathrm{SI}\right|^{\mathrm{a}}\end{array}$ & $\begin{array}{l}\text { Reproduction } \\
\text { number (R) }\end{array}$ & $95 \% \mathrm{Cl}^{\mathrm{b}}$ & $\begin{array}{l}\text { Basic or } \\
\text { effective }\end{array}$ & Case definition & Reference & $\begin{array}{l}\text { Year } \\
\text { published }\end{array}$ \\
\hline $\begin{array}{l}1889- \\
1890\end{array}$ & H3N8? & USA \& Europe & 2.6 & 2.10 & $1.9-2.4$ & Basic & Unconfirmed deaths & [101] & 2010 \\
\hline $\begin{array}{l}1948- \\
1949\end{array}$ & $\mathrm{H} 1 \mathrm{~N} 1$ & Canada & 4.1 & 1.30 & & Effective & Unconfirmed illness & [25] & 2006 \\
\hline $\begin{array}{l}1949- \\
1950\end{array}$ & $\mathrm{H} 1 \mathrm{~N} 1$ & Canada & 4.1 & 1.50 & & Effective & Unconfirmed illness & {$[25]$} & 2006 \\
\hline $\begin{array}{l}1950- \\
1951\end{array}$ & H1N1 & Canada \& UK & 4.1 & 2.00 & $1.9-2.5$ & Effective & Unconfirmed deaths & {$[25]$} & 2006 \\
\hline $\begin{array}{l}1958- \\
1973\end{array}$ & $\begin{array}{l}\mathrm{H} 2 \mathrm{~N} 2 ; \\
\mathrm{H} 3 \mathrm{~N} 2 ; \mathrm{B}\end{array}$ & $\begin{array}{l}\text { United } \\
\text { Kingdom }\end{array}$ & 4.48 & $3.9-7.1$ & & Effective & Unconfirmed illness & [102] & 1979 \\
\hline $\begin{array}{l}1972- \\
2002\end{array}$ & $\begin{array}{l}\text { H1N1/ } \\
\text { H3N2/B }\end{array}$ & Australia & 5.5 & 1.30 & & Effective & Unconfirmed deaths & [103] & 2008 \\
\hline $\begin{array}{l}1972- \\
2002\end{array}$ & $\begin{array}{l}\text { H1N1/ } \\
\text { H3N2/B }\end{array}$ & France & 5.5 & 1.30 & & Effective & Unconfirmed deaths & [103] & 2008 \\
\hline $\begin{array}{l}1972- \\
2002\end{array}$ & $\begin{array}{l}\text { H1N1/ } \\
\text { H3N2/B }\end{array}$ & USA & 5.5 & 1.30 & & Effective & Unconfirmed deaths & [103] & 2008 \\
\hline $\begin{array}{l}1972- \\
2002\end{array}$ & $\begin{array}{l}\text { H1N1/ } \\
\text { H3N2/B }\end{array}$ & $\begin{array}{l}\text { USA; France; } \\
\text { Australia }\end{array}$ & 5.5 & 1.30 & $1.2-1.4$ & Effective & Unconfirmed deaths & [103] & 2008 \\
\hline $\begin{array}{l}1975- \\
2004\end{array}$ & $\begin{array}{l}\text { H1N1/ } \\
\text { H3N2/B }\end{array}$ & Norway & 6 & $1.06-1.69$ & & Effective & Unconfirmed deaths & [104] & 2010 \\
\hline $\begin{array}{l}1976- \\
1981\end{array}$ & $\begin{array}{l}\text { H1N1/ } \\
\text { H3N2/B }\end{array}$ & USA & 2.6 & 1.70 & & Basic & $\begin{array}{l}\text { Serology confirmed } \\
\text { infection }\end{array}$ & [10] & 2006 \\
\hline $\begin{array}{l}1976- \\
1981\end{array}$ & $\begin{array}{l}\text { H1N1/ } \\
\text { H3N2/B }\end{array}$ & USA & 4.1 & 1.16 & & Basic & $\begin{array}{l}\text { Serology confirmed } \\
\text { infection }\end{array}$ & [105] & 2000 \\
\hline $\begin{array}{l}1977- \\
1978\end{array}$ & $\mathrm{H} 1 \mathrm{~N} 1$ & $\begin{array}{l}\text { United } \\
\text { Kingdom }\end{array}$ & 2.2 & 4.38 & & Basic & Unconfirmed illness & {$[106]$} & 2005 \\
\hline $\begin{array}{l}1977- \\
1978\end{array}$ & $\mathrm{H} 1 \mathrm{~N} 1$ & $\begin{array}{l}\text { United } \\
\text { Kingdom }\end{array}$ & 3 & 21.00 & & Basic & Unconfirmed illness & [13] & 2004 \\
\hline $\begin{array}{l}1977- \\
1978\end{array}$ & $\mathrm{H} 1 \mathrm{~N} 1$ & $\begin{array}{l}\text { United } \\
\text { Kingdom }\end{array}$ & 4.70 & 16.90 & & Basic & Unconfirmed illness & {$[106]$} & 2005 \\
\hline $\begin{array}{l}1984- \\
1985\end{array}$ & $\begin{array}{l}\text { H1N1/ } \\
\text { H3N2 }\end{array}$ & France & 2.49 & 1.37 & & Effective & Unconfirmed illness & [107] & 1988 \\
\hline $\begin{array}{l}1985- \\
2005\end{array}$ & $\begin{array}{l}\text { H1N1/ } \\
\text { H3N2/B }\end{array}$ & $\begin{array}{l}\text { United } \\
\text { Kingdom }\end{array}$ & 2.2 & $1.6-2.1$ & & Basic & Unconfirmed illness & [108] & 2010 \\
\hline $\begin{array}{l}1985- \\
2005\end{array}$ & $\begin{array}{l}\text { H1N1/ } \\
\text { H3N2/B }\end{array}$ & $\begin{array}{l}\text { United } \\
\text { Kingdom }\end{array}$ & 2.7 & $1.6-2.5$ & & Basic & Unconfirmed illness & [109] & 2012 \\
\hline $\begin{array}{l}1985- \\
2006\end{array}$ & $\begin{array}{l}\text { H1N1/ } \\
\text { H3N2/B }\end{array}$ & France & 2.4 & $1.4-1.7$ & $1.3-1.8$ & Basic & Unconfirmed illness & [110] & 2008 \\
\hline $\begin{array}{l}1996- \\
2006\end{array}$ & $\begin{array}{l}\text { H1N1/ } \\
\text { H3N2/B }\end{array}$ & Brazil & 3 & 1.03 & $1.02-1.04$ & Effective & Unconfirmed deaths & [111] & 2010 \\
\hline $\begin{array}{l}1998- \\
1999\end{array}$ & $\mathrm{H} 3 \mathrm{~N} 2$ & Israel & 3 & 1.14 & & Effective & Unconfirmed illness & [112] & 2011 \\
\hline $\begin{array}{l}1998- \\
1999\end{array}$ & $\mathrm{H} 3 \mathrm{~N} 2$ & Israel & 3 & 1.16 & & Effective & Unconfirmed illness & [112] & 2011 \\
\hline $\begin{array}{l}1998- \\
1999\end{array}$ & $\mathrm{H} 3 \mathrm{~N} 2$ & USA & 3 & 1.18 & $1.05-1.25$ & Effective & $\begin{array}{l}\text { Laboratory confirmed } \\
\text { illness }\end{array}$ & [113] & 2009 \\
\hline $\begin{array}{l}1998- \\
2009\end{array}$ & $\begin{array}{l}\text { H1N1/ } \\
\text { H3N2/B }\end{array}$ & Israel & 2.5 & $1.17-1.62$ & & Effective & Unconfirmed illness & [114] & 2012 \\
\hline $\begin{array}{l}1999- \\
2000\end{array}$ & $\mathrm{H} 3 \mathrm{~N} 2$ & Israel & 3 & 1.16 & & Effective & Unconfirmed illness & [112] & 2011 \\
\hline $\begin{array}{l}1999- \\
2000\end{array}$ & $\mathrm{H} 3 \mathrm{~N} 2$ & Israel & 3 & 1.18 & & Effective & Unconfirmed illness & [112] & 2011 \\
\hline
\end{tabular}


Table 5 Reproduction numbers from seasonal influenza epidemics (Continued)

\begin{tabular}{|c|c|c|c|c|c|c|c|c|c|}
\hline $\begin{array}{l}1999- \\
2006\end{array}$ & $\begin{array}{l}\text { Seasonal } \\
\text { H1N1 }\end{array}$ & Taiwan & 2 & 1.19 & $0.76-1.86$ & Basic & $\begin{array}{l}\text { Confirmed and } \\
\text { unconfirmed illness }\end{array}$ & [115] & 2010 \\
\hline $\begin{array}{l}1999- \\
2006\end{array}$ & $\mathrm{H} 3 \mathrm{~N} 2$ & Taiwan & 3 & 1.41 & $0.92-2.19$ & Basic & $\begin{array}{l}\text { Confirmed and } \\
\text { unconfirmed illness }\end{array}$ & [115] & 2010 \\
\hline $\begin{array}{l}1999- \\
2006\end{array}$ & B & Taiwan & 3 & 1.07 & $0.69-1.69$ & Basic & $\begin{array}{l}\text { Confirmed and } \\
\text { unconfirmed illness }\end{array}$ & [115] & 2010 \\
\hline $\begin{array}{l}2000- \\
2001\end{array}$ & $\mathrm{H} 1 \mathrm{~N} 1$ & Israel & 3 & 1.12 & & Effective & Unconfirmed illness & [112] & 2011 \\
\hline $\begin{array}{l}2000- \\
2009\end{array}$ & $\begin{array}{l}\text { H1N1/ } \\
\text { H3N2/B }\end{array}$ & Italy & 4 & $1.17-1.36$ & & Effective & Unconfirmed illness & [116] & 2012 \\
\hline $\begin{array}{l}2001- \\
2002\end{array}$ & $\mathrm{H} 3 \mathrm{~N} 2$ & Israel & 3 & 1.25 & & Effective & Unconfirmed illness & [112] & 2011 \\
\hline $\begin{array}{l}2001- \\
2002\end{array}$ & $\mathrm{H} 3 \mathrm{~N} 2$ & Israel & 3 & 1.27 & & Effective & Unconfirmed illness & [112] & 2011 \\
\hline $\begin{array}{l}2003- \\
2004\end{array}$ & $\mathrm{H} 3 \mathrm{~N} 2$ & Israel & 3 & 1.19 & & Effective & Unconfirmed illness & [112] & 2011 \\
\hline $\begin{array}{l}2003- \\
2004\end{array}$ & $\mathrm{H} 3 \mathrm{~N} 2$ & Israel & 3 & 1.21 & & Effective & Unconfirmed illness & [112] & 2011 \\
\hline $\begin{array}{l}2003- \\
2004\end{array}$ & $\mathrm{H} 3 \mathrm{~N} 2$ & Switzerland & 2.6 & $1.2-1.3$ & & Effective & Unconfirmed illness & [117] & 2011 \\
\hline $\begin{array}{l}2004- \\
2005\end{array}$ & $\mathrm{H} 3 \mathrm{~N} 2$ & Israel & 3 & 1.25 & & Effective & Unconfirmed illness & [112] & 2011 \\
\hline $\begin{array}{l}2004- \\
2005\end{array}$ & $\mathrm{H} 3 \mathrm{~N} 2$ & Israel & 3 & 1.25 & & Effective & Unconfirmed illness & [112] & 2011 \\
\hline $\begin{array}{l}2004- \\
2005\end{array}$ & unspecified & Taiwan & 4.1 & 1.00 & & Effective & Unconfirmed deaths & [118] & 2010 \\
\hline $\begin{array}{l}2004- \\
2005\end{array}$ & $\mathrm{H} 3 \mathrm{~N} 2$ & USA & 7 & 1.20 & $1.1-1.3$ & Effective & $\begin{array}{l}\text { Laboratory confirmed } \\
\text { illness }\end{array}$ & [119] & 2008 \\
\hline $\begin{array}{l}2006- \\
2007\end{array}$ & $\mathrm{H} 3 \mathrm{~N} 2$ & Israel & 3 & 1.28 & & Effective & Unconfirmed illness & [112] & 2011 \\
\hline $\begin{array}{l}2006- \\
2007\end{array}$ & $\mathrm{H} 3 \mathrm{~N} 2$ & Israel & 3 & 1.33 & & Effective & Unconfirmed illness & [112] & 2011 \\
\hline $\begin{array}{l}2007- \\
2008\end{array}$ & $\mathrm{H} 3 \mathrm{~N} 2$ & Israel & 3 & 1.25 & & Effective & Unconfirmed illness & [112] & 2011 \\
\hline $\begin{array}{l}2007- \\
2008\end{array}$ & $\mathrm{H} 3 \mathrm{~N} 2$ & Israel & 3 & 1.29 & & Effective & Unconfirmed illness & [112] & 2011 \\
\hline $2011 / 12$ & $\mathrm{H} 1 \mathrm{~N} 1$ & Mexico & 3 & 1.20 & & Effective & $\begin{array}{l}\text { Laboratory confirmed } \\
\text { hospitalizations }\end{array}$ & [120] & 2012 \\
\hline $2011 / 12$ & $\mathrm{H} 1 \mathrm{~N} 1$ & Mexico & 3 & 1.20 & & Effective & $\begin{array}{l}\text { Laboratory confirmed } \\
\text { hospitalizations }\end{array}$ & [121] & 2012 \\
\hline $2011 / 12$ & $\mathrm{H} 1 \mathrm{~N} 1$ & Mexico & 4 & 1.30 & & Effective & $\begin{array}{l}\text { Laboratory confirmed } \\
\text { hospitalizations }\end{array}$ & [121] & 2012 \\
\hline
\end{tabular}

${ }^{a}$ The generation time (GT) or serial interval (SI) assumed in the reproduction number estimation

${ }^{\mathrm{b}}$ Confidence interval

$\mathrm{NR}=$ Not reported

This table is also available as a .csv file as Additional file 5 .

(26\%). The mean generation time or serial interval used in the calculations to determine $\mathrm{R}$ had a median value of 2.8 days, and the mean ranged from 1.9-7 days (Table 4). A majority of the studies included for the 2009 pandemic relied on either laboratory-confirmed illnesses (71\%) or unconfirmed illnesses (24\%) to identify those ill (Table 4).

\section{Seasonal influenza}

Seasonal influenza causes sustained epidemics in the nontropical areas of the Northern Hemisphere and Southern Hemisphere during their respective late fall to early spring months. Epidemics in the tropical regions occur sporadically but can be associated with the rainy season [1]. The 
Table 6 Reproduction numbers from novel influenza outbreaks

\begin{tabular}{|c|c|c|c|c|c|c|c|c|c|}
\hline Year(s) & Subtype & $\begin{array}{c}\text { Study } \\
\text { Population }\end{array}$ & $\begin{array}{l}\text { Mean } \\
\mathrm{GT} / \mathrm{SI} \mathbf{I}^{\mathrm{a}}\end{array}$ & $\begin{array}{c}\text { Reproduction } \\
\text { number (R) }\end{array}$ & $95 \% \mathrm{Cl}^{\mathrm{b}}$ & $\begin{array}{l}\text { Basic or } \\
\text { Effective }\end{array}$ & Case definition & Reference & $\begin{array}{c}\text { Year } \\
\text { Published }\end{array}$ \\
\hline 1976 & H1N1 & New Jersey & 1.9 & 1.20 & $1.1-1.4$ & Basic & $\begin{array}{c}\text { Serologically confirmed } \\
\text { illness }\end{array}$ & [122] & 2007 \\
\hline $\begin{array}{l}2004- \\
2006\end{array}$ & $\mathrm{H} 5 \mathrm{~N} 1$ & Vietnam & 7 & 0.00 & $0-0.42$ & Effective & $\begin{array}{l}\text { Laboratory confirmed } \\
\text { illness }\end{array}$ & [119] & 2008 \\
\hline $\begin{array}{l}2004- \\
2006\end{array}$ & $\mathrm{H} 5 \mathrm{~N} 1$ & Indonesia & 7 & 0.00 & $0-0$ & Effective & $\begin{array}{l}\text { Laboratory confirmed } \\
\text { illness }\end{array}$ & [119] & 2008 \\
\hline 2005 & $\mathrm{H} 5 \mathrm{~N} 1$ & Turkey & 9.5 & $<1$ & & Basic & $\begin{array}{l}\text { Laboratory confirmed } \\
\text { illness }\end{array}$ & [123] & 2007 \\
\hline $\begin{array}{l}2005- \\
2009\end{array}$ & H5N1 & Indonesia & 6 & $0.1-0.25$ & $0-0.4$ & Effective & $\begin{array}{l}\text { Laboratory confirmed } \\
\text { illness }\end{array}$ & [124] & 2012 \\
\hline 2006 & $\mathrm{H} 5 \mathrm{~N} 1$ & Indonesia & 9.5 & 1.14 & $0.61-2.14$ & Basic & $\begin{array}{l}\text { Laboratory confirmed } \\
\text { illness }\end{array}$ & [123] & 2007 \\
\hline
\end{tabular}

Median reproduction number for novel influenza outbreaks: 0.34; Interquartile range 0.05-0.98

${ }^{a}$ The generation time (GT) or serial interval (SI) assumed in the reproduction number estimation. $\mathrm{NR}=$ Not reported

${ }^{\mathrm{b}}$ Confidence interval.

This table is also available as a .csv file as Additional file 6.

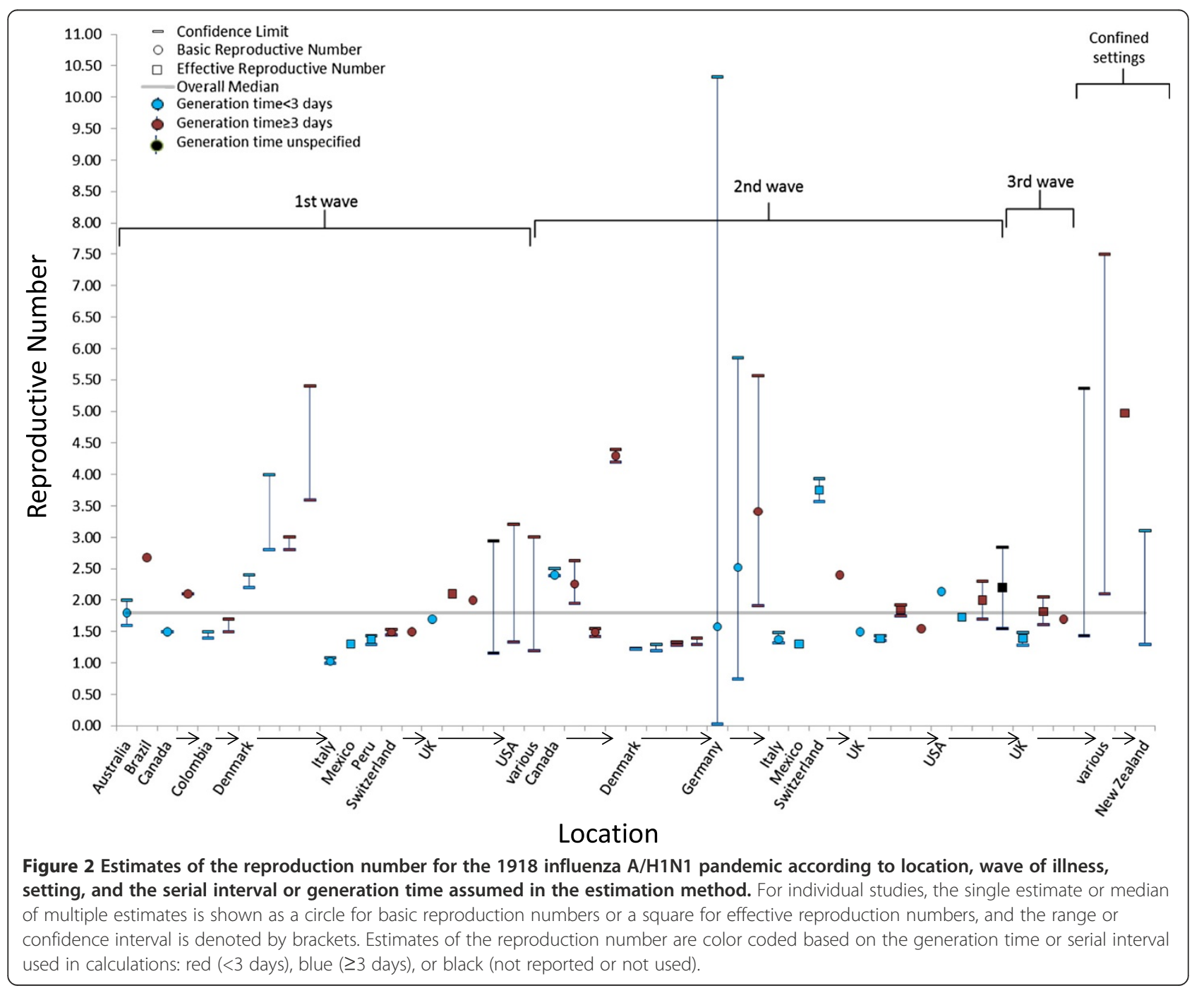




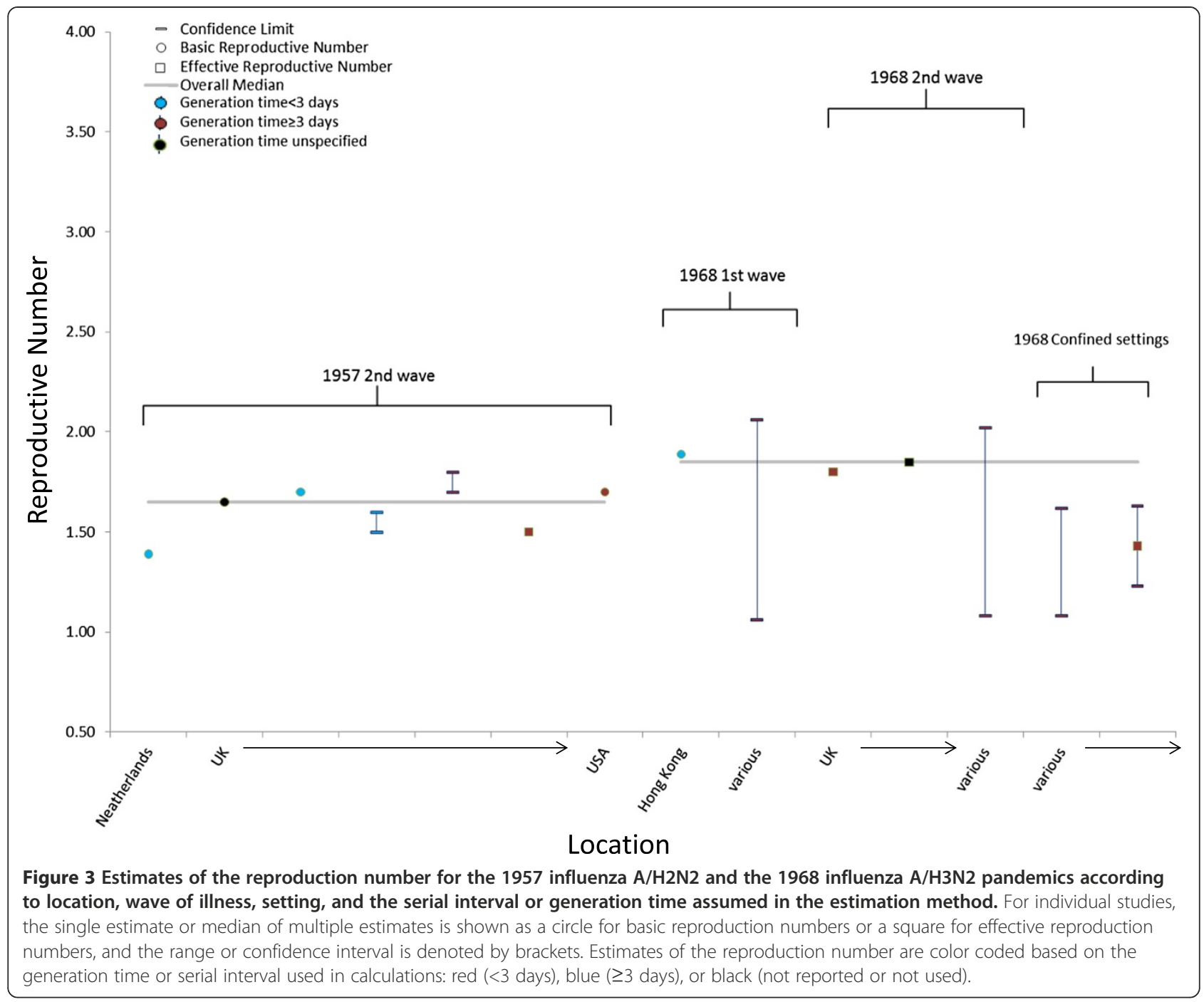

mortality burden from influenza varies by season, and from 1976-2007, estimates of annual influenza-associated deaths in the United States from respiratory and circulatory causes ranged from 3,000 to 49,000 [3].

Twenty-four studies reported 47 separate seasonal epidemic values of $\mathrm{R}$ (Table 5; Figure 5). The median point estimate of $\mathrm{R}$ in the community setting for seasonal influenza was 1.27 (IQR: 1.19-1.37) while a higher median $R$ value $(R=16.9)$ was reported in a British boarding school during the 1977-78 influenza season (Table 5). R values for seasons where $\mathrm{H} 3 \mathrm{~N} 2(\mathrm{R}=1.25$; IQR: $1.18-$ 1.27) or $\mathrm{H} 1 \mathrm{~N} 1$ ( $\mathrm{R}=1.25$; IQR: $1.18-1.35)$ predominated were equivalent (Table 5).

A majority of seasonal influenza values for $\mathrm{R}$ were calculated for populations in Israel (35\%), Europe (25\%), and North America (21\%). The mean generation time or serial interval used in the calculations to determine $\mathrm{R}$ had a median value of 3.0 days, and the mean ranged from 2.0-7.0 days (Table 5). A majority of the studies included for seasonal influenza relied on unconfirmed illnesses or deaths (79\%); the reminder relied on either laboratory-confirmed illnesses or hospitalizations or serologically-confirmed infections (Table 5).

\section{Human infections with non-human influenza viruses}

Human infections with novel or non-human influenza viruses (also known as zoonotic influenza viruses) are rare but can result in a pandemic if sustained personto-person transmission occurs and the population has little or no pre-existing population immunity to the virus. Therefore, instances of infection with nonhuman influenza viruses are investigated thoroughly to assess the transmissibility of the virus. The largest number of novel influenza cases at the time of this review was from the ongoing influenza A/H5N1 outbreak centered in Southeast Asia and the Middle 


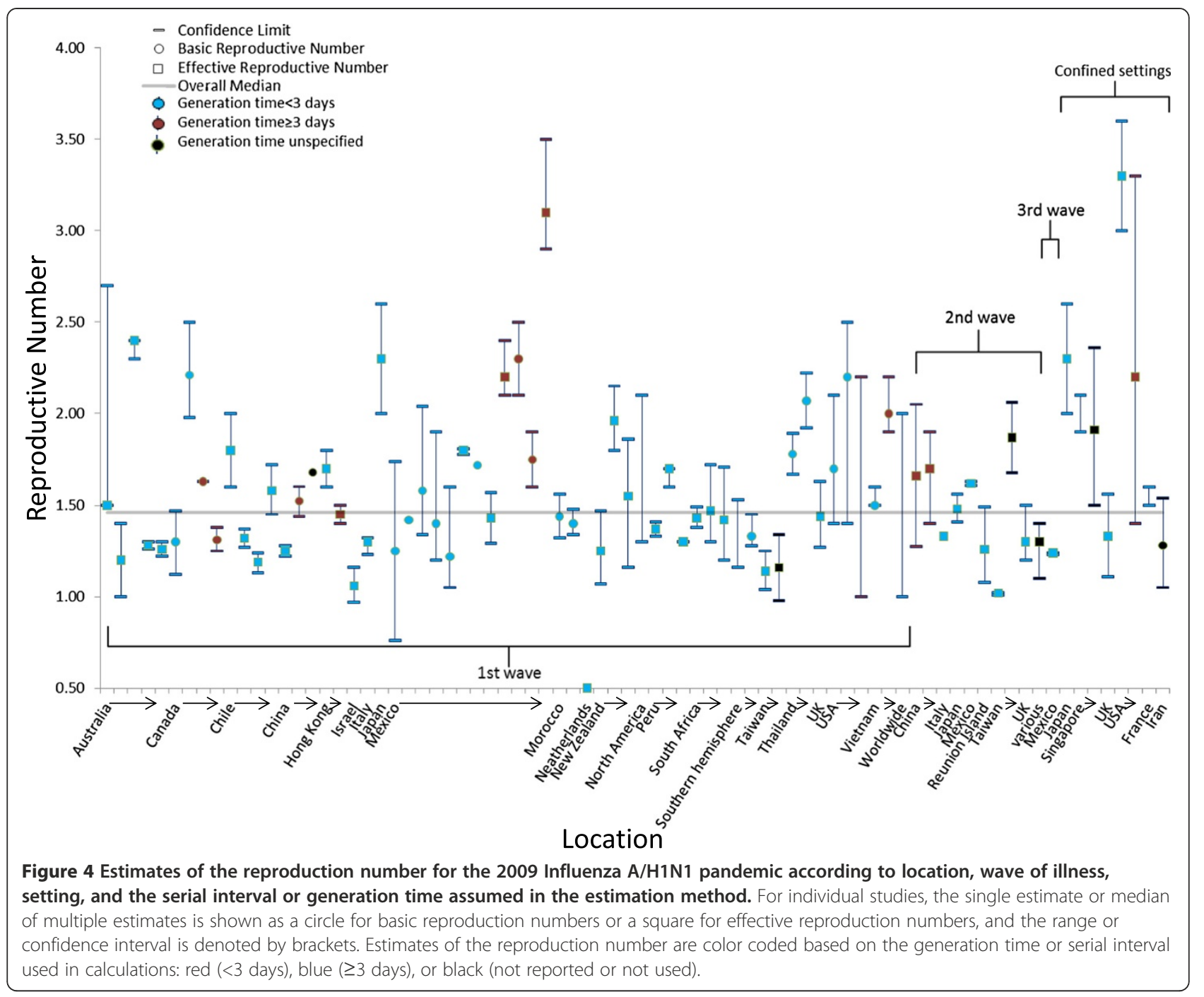

East. From January, 1, 2003 to February 15, 2013, 620 laboratory-confirmed cases have been reported to the WHO, of which 367 have died [127]. Another large outbreak of novel influenza occurred in 1976 in Fort Dix, New Jersey, which was caused by an influenza A/H1N1 virus similar to those found circulating in swine [122].

Four studies estimated the values of $\mathrm{R}$ for the $\mathrm{A} / \mathrm{H} 5 \mathrm{~N} 1$ and A/H1N1 outbreaks (Table 6). Four out of six estimates $(67 \%)$ of $\mathrm{R}$ were less than one, and the highest $R$ estimate $(R=1.2)$ was for the $1976 \mathrm{~A} / \mathrm{H} 1 \mathrm{~N} 1$ outbreak in a New Jersey military camp (a confined setting) (Table 6).

A majority of novel A virus $\mathrm{R}$ values were calculated for populations in Southeast Asia (67\%), indicative of where the bulk of $\mathrm{A} / \mathrm{H} 5 \mathrm{~N} 1$ bird-to-human transmission occurs. The mean generation time or serial interval used in the calculations to determine $R$ had a median value of 7.0 days, and the mean ranged from 1.9-9.5 days (Table 6). All studies relied on either laboratory-confirmed illness or serological-confirmed infection (Table 6).

\section{Discussion}

In this review, the median $\mathrm{R}$ values reported for the four pandemics and seasonal influenza varied between 1.271.8 while $\mathrm{R}$ values for novel influenza were generally below 1. We found the highest median reproduction number associated with the 1918 and the 1968 influenza pandemics (both 1.8), followed by the 1957 pandemic (1.65), the 2009 pandemic (1.46), seasonal influenza epidemics (1.27), and novel influenza outbreaks. A majority of $\mathrm{R}$ values published were for either the 1918 pandemic or the 2009 pandemic; the 1957 and 1968 pandemics had the fewest published studies. Researchers calculated values for $\mathrm{R}$ for a variety of locations and utilized many 


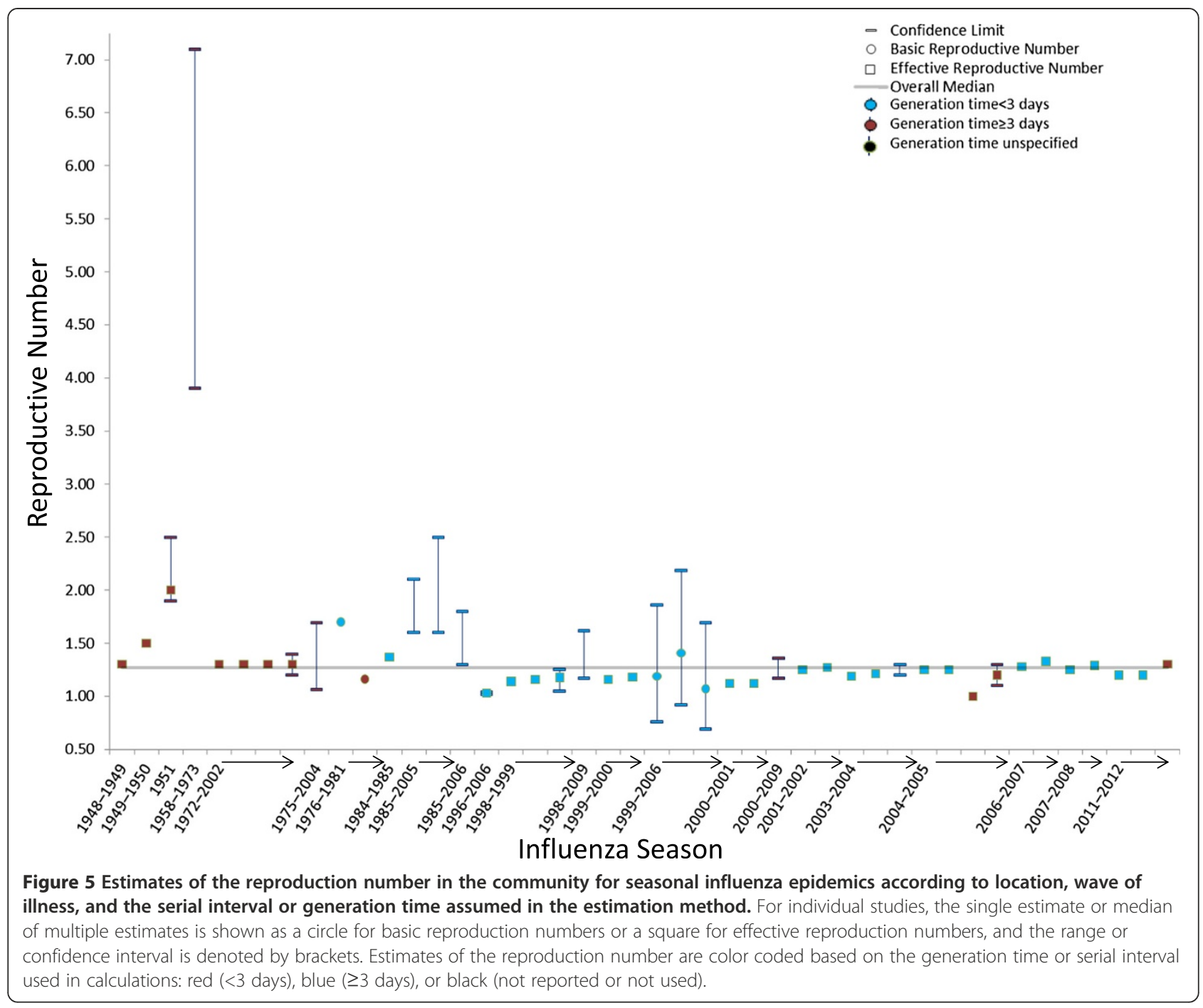

different case definitions, ascertainment methods, and assumptions about the generation time or serial interval.

The approximate basic reproductive numbers for some common infectious diseases range from 12-18 for measles, 12-17 for pertussis, and 4-7 for mumps, polio, rubella, and smallpox [12]. These values are much higher than what has been reported for influenza, and most $\mathrm{R}$ values reported in this review ranged from 1.0-2.0. However, the overall clinical attack rate and peak daily incidence of an outbreak, which measures the potential burden on healthcare services and school and workplace absenteeism, are very sensitive to changes in the value of $\mathrm{R}$ within this range. Past research utilizing a number of assumptions on the symptomatic ratio, contact patterns, and seeding has estimated that the cumulative clinical attack rates for a pandemic when $\mathrm{R}=1.3$ ranged from $15 \%-21 \%$ and increased to $34 \%-42 \%$ for $\mathrm{R}=2.0[10,11]$. Similarly, the peak daily attack rate is $0.5 \%$ for $\mathrm{R}=1.3$ and $2.2 \%$ for $\mathrm{R}=2.0$ [10]. Therefore, with only an absolute difference in $\mathrm{R}$ of 0.7 , the clinical attack rates in these studies more than doubled and the peak daily incidence more than quadrupled.

Differences in the value of $\mathrm{R}$ within this range also affect the evaluation of potential mitigation strategies (e. g., school closures, vaccination, household isolation) for influenza pandemics. Analysis of strategies to mitigate an influenza pandemic have found that the effectiveness of non-travel-related control policies, such as school closures, household quarantine, and vaccination, would decrease as the value of $\mathrm{R}$ increases from 1.0 to 2.0 [10]. The success of various vaccination strategies would also be more likely for values of $\mathrm{R}<1.7[10,11]$. Therefore, the small variations in pandemic $R$ estimates found in this analysis can have important implications for the overall impact and success of mitigation efforts for an influenza pandemic. This finding highlights the importance 
of making precise estimates of $\mathrm{R}$ early in a pandemic. Further research should focus on refining methods that allow for early, robust estimates of R.

The results of this analysis reinforce the idea that $\mathrm{R}$ is a measure that captures the transmissibility of an influenza virus in the population under study and is not an intrinsic value. The inputs for its calculation can include the population contact rate, the probability of infection per contact, the duration of illness, and the percentage of the population that is susceptible which is affected by the characteristics of the population under study. Therefore, the variations in the value for $\mathrm{R}$ for the same pandemic or seasonal outbreak are expected and may be due to the underlying social and socio-demographic factors of the population studied, public health interventions, and geographical or climatic factors of the location. These variations include the percentage of the source's population under 18 years old; differences in contact patterns between age groups, which vary by country $[128,129]$; and differences in population susceptibility profiles, which varied by age group for the 2009 pandemic [130]. Another important factor that may contribute to the variation is the season from which data used to estimate $\mathrm{R}$ is collected. While the effect of weather on the transmissibility of influenza has not been fully explored, some studies have shown that the level of absolute humidity is inversely correlated with influenza transmissibility [131,132]. Therefore, estimates of $\mathrm{R}$ should be interpreted in the context of the population under study and the season in which data was collected and direct comparisons of $\mathrm{R}$ between populations should be undertaken with caution.

Variations in the estimated values of $\mathrm{R}$ may also be driven by changes in surveillance intensity in the same country over time. If a country suddenly improves its surveillance system in response to a pandemic and is better able to identify cases, then the number of cases being reported will increase, even though the actual number of cases occurring will not have changed. This increase in the reported number of cases may increase the estimated $R$ as the growth rate of the outbreak will increase [86]. Conversely, the value of $\mathrm{R}$ could be artificially lowered if countries implement changes in surveillance practices that result in a lower number of identified cases, such as reducing screening recommendations, or have their surveillance systems overwhelmed. This effect was seen in the United States during the 2009 pandemic, when influenza testing for every case became unfeasible and testing recommendations were changed [4].

One of the more important methodological assumptions that can have a large impact on the estimated value of $\mathrm{R}$ is the length of the serial interval or generation time used during the estimation of R. Longer serial intervals have previously been associated with higher estimates of
$\mathrm{R}$ when compared to estimates from the same dataset using shorter serial intervals [9]. In this analysis, estimates of $\mathrm{R}$ from the 1918, 1957, and 1968 pandemics utilized higher serial interval values than were used for the 2009 pandemic or for seasonal influenza. Additionally, higher values of $\mathrm{R}$ from the 2009 pandemic often were estimated using a generation time or serial interval of 3 days or more (Figure 4). Therefore, the estimates of $\mathrm{R}$ included in this analysis should be interpreted in the context of the serial intervals or generation times used in the estimation method. Like R, the values for the generation time or the serial interval can vary by the source population. Therefore, researchers estimating the values of $\mathrm{R}$ should strive to use standard estimates of the serial interval or generation time for influenza or at least include common values in a sensitivity analysis. This will help with the comparability of $\mathrm{R}$ values across studies and may aid in the correct interpretation of $\mathrm{R}$ estimates. An additional way in which estimates of $R$ may be biased up or down lies in the choice of estimation procedure itself. Chowell et al. showed that estimates of $\mathrm{R}$ obtained using simple epidemic mathematical models varied considerably as the model increased in complexity (e.g. the addition of a period of infection latency or an agestructured population) [35].

Although we found no difference in the value of $\mathrm{R}$ for studies using confirmed cases versus unconfirmed cases in the estimation method, the trade-off between the accuracy of the less specific but more efficient and cost effective syndromic data compared to laboratory-confirmed influenza infections is unknown. The incubation periods of non-influenza respiratory pathogens that co-circulate with influenza (e.g. respiratory syncytial virus or rhinovirus) range from a median of 1.9-5.6 days; estimates of $R$ for influenza could either be overestimated or underestimated during periods of co-circulation, depending on the intensity and identity of the co-circulating respiratory pathogen [87]. Future research should focus on estimation of $\mathrm{R}$ using laboratory-confirmed cases and hospitalizations and should provide estimates from syndromic data for comparison.

Most studies included in this analysis focused on 1918 or the 2009 pandemic. Only a small number of estimates of the reproduction number have been reported for the two other pandemics of the $20^{\text {th }}$ century (1957 and 1968). As a consequence, there is still insufficient information to fully clarify the transmission dynamics of the 1957 and 1968 pandemics. Because historical data are available for these pandemics, future research should focus on estimations of R for the 1957 and 1968 pandemics to better understand the characteristics of these pandemics.

This study generally found higher reproduction numbers for confined settings, such as schools, military 
bases, or night clubs, except for estimates from the 1968 pandemic. Because confined settings increase the intensity of transmission by increasing contact rates among those ill and well, the values of $\mathrm{R}$ presented for outbreaks in confined settings are likely to be much higher than values of $\mathrm{R}$ estimated for the community and should be interpreted accordingly. While the estimation of $\mathrm{R}$ in confined settings may be useful for the assessment of the upper bounds of transmissibility, its value is not directly comparable to estimates of $\mathrm{R}$ made in the community setting.

This review found, with one exception, a high degree of consistency in the estimated values of $\mathrm{R}$ for seasonal influenza epidemics. The only notable exception was the extremely high $\mathrm{R}$ values estimated for an outbreak of influenza A (H1N1) in 1978 at a small British boarding school with 763 male students aged 10-18 who were mostly full boarders [133]. The results of this analysis suggest that the extreme $\mathrm{R}$ values reported for this outbreak are not typical of seasonal or pandemic influenza and instead may be the result of the lack of pre-existing immunity among the students to the strain of influenza A (H1N1) that caused the outbreak, the extremely high contact rates likely among a group of boarded students, or a study artifact related to the small number of students in the study population $[13,106,133]$. Additionally, the median $R$ value of seasonal influenza $(R=1.27)$ is well below the median values seen during the four pandemics examined in this report. The consistency of seasonal $\mathrm{R}$ values is even more remarkable given the wide variety of estimation methods, data sources, and assumptions used in the studies included here. However, the majorities of seasonal influenza estimates were from a small number of countries. Estimates of $\mathrm{R}$ from countries in Africa, Asia, and South America are also needed to determine if values of $\mathrm{R}$ for seasonal influenza epidemics are affected by geographic and social factors.

This systematic review is subject to at least three limitations. First, we combined estimates for the basic and effective reproductive numbers when presenting the median estimates in this study. Even though these values measure transmission in populations with differing levels of underlying population immunity, some papers included in this review did not clearly differentiate between basic and effective reproductive numbers or state the required population immunity assumptions when reporting basic reproductive numbers. Therefore, we choose to present summary values for the basic and effective reproductive numbers together to simplify the results. The tables include whether the reproductive number estimate was reported as basic or effective for each study. Second, we did not assess included studies for the type or quality of their methodology or the risk of study bias. Finally, we only included published estimates of the reproductive number, which may not be representative of unpublished reproductive number values.

\section{Conclusions}

In this review, we explored the ranges and uncertainty of the values of $\mathrm{R}$ estimated for seasonal, pandemic, and novel influenza. We found that values of $\mathrm{R}$ changed over the course of a pandemic but the effect of the waves varied. The value of $R$ is not constant and may be affected by mitigation strategies, the season, and the population under study. The values of $\mathrm{R}$ found in this analysis represent the difference between a pandemic that is controllable with less intensive mitigation strategies and would cause moderate amounts of illness to a pandemic that would require very intensive mitigation strategies and would cause greater amounts of illness. Continued monitoring of $\mathrm{R}$ during outbreaks of human infections with non-human influenza viruses and in various settings throughout future pandemics will be required to fully understand the effects of mitigation, geography, and season.

\section{Additional files}

Additional file 1: Reproduction Numbers from the 1918 Influenza A/H1N1 Pandemic.

Additional file 2: Reproduction Numbers from the 1957 Influenza A/H2N2 Pandemic.

Additional file 3: Reproduction Numbers from the 1968 Influenza A/H3N2 Pandemic.

Additional file 4: Reproduction Numbers from the 2009 Influenza A/H1N1 Pandemic.

Additional file 5: Reproduction Numbers from Seasonal Influenza Epidemics.

Additional file 6: Reproduction Numbers from Novel Influenza Outbreaks.

\section{Competing interest}

The authors declare that they have no financial or non-financial competing interests with the publication of this manuscript.

\section{Authors' contributions}

MB led the data collection, analysis, and the writing of the article. SC led the editing of the article and assisted with data interpretation. CR and MG contributed significantly to data interpretation and reviewed multiple drafts of the article. LF contributed to the design of the study, data interpretation, and reviewed multiple drafts of the article. All authors read and approved the final manuscript.

\section{Acknowledgements}

We are particularly grateful for the assistance in the preparation and editing of the manuscript given by Alejandro Perez and Dr. Claudia Campbell.

\section{Disclaimer}

The findings and conclusions in this report are those of the authors and do not necessarily represent the official position of the Centers for Disease Control and Prevention. 


\section{Author details}

'Epidemiology and Prevention Branch, Influenza Division, National Center for Immunization and Respiratory Diseases, Centers for Disease Control and Prevention, 1600 Clifton Road NE MS A-32, Atlanta 30333, Georgia.

${ }^{2}$ Mathematical Modelling of Infectious Diseases Unit, Institut Pasteur, Paris, France. ${ }^{3}$ National Center for Immunization and Respiratory Diseases, CDC, Atlanta, Georgia.

Received: 11 April 2014 Accepted: 28 August 2014

Published: 4 September 2014

\section{References}

1. Cox NJ, Subbarao K: Global epidemiology of influenza: past and present. Annu Rev Med 2000, 51:407-421.

2. Sullivan KM, Monto AS, Longini IM Jr: Estimates of the US health impact of influenza. Am J Public Health 1993, 83(12):1712-1716.

3. Centers for Disease Control and Prevention: Estimates of deaths associated with seasonal influenza -- United States, 1976-2007. MMWR Morb Mortal Wkly Rep 2010, 59(33):1057-1062.

4. Jhung MA, Swerdlow D, Olsen SJ, Jernigan D, Biggerstaff M, Kamimoto L, Kniss K, Reed C, Fry A, Brammer L, Gindler J, Gregg WJ, Bresee J, Finelli L: Epidemiology of 2009 pandemic influenza A (H1N1) in the United States. Clin Infect Dis 2011, 52(Suppl 1):S13-S26.

5. Reed C, Biggerstaff $M$, Finelli L, Koonin LM, Beauvais D, Uzicanin A, Plummer A, Bresee J, Redd SC, Jernigan DB: Novel framework for assessing epidemiologic effects of influenza epidemics and pandemics. Emerg Infect Dis 2013, 19(1):85-91.

6. Anderson RM, May RM: Infectious diseases of humans : dynamics and control. Oxford. New York: Oxford University Press; 1991

7. Heffernan JM, Smith RJ, Wahl LM: Perspectives on the basic reproductive ratio. Journal of the Royal Society, Interface / the Royal Society 2005, 2(4):281-293.

8. Li J, Blakeley D, Smith RJ: The failure of Ro. Computational and mathematical methods in medicine 2011, 2011:527610.

9. Boelle PY, Ansart S, Cori A, Valleron AJ: Transmission parameters of the A/H1N1 (2009) influenza virus pandemic: a review. Influenza Other Respi Viruses 2011, 5(5):306-316.

10. Ferguson NM, Cummings DA, Fraser C, Cajka JC, Cooley PC, Burke DS: Strategies for mitigating an influenza pandemic. Nature 2006, 442(7101):448-452

11. Yang $Y$, Sugimoto JD, Halloran ME, Basta NE, Chao DL, Matrajt L, Potter G, Kenah E, Longini IM Jr: The transmissibility and control of pandemic influenza A (H1N1) virus. Science (New York, NY) 2009, 326(5953):729-733.

12. Fine PE: Herd immunity: history, theory, practice. Epidemiol Rev 1993, 15(2):265-302.

13. Fraser $C$, Riley $S$, Anderson RM, Ferguson NM: Factors that make an infectious disease outbreak controllable. Proc Natl Acad Sci U S A 2004, 101(16):6146-6151.

14. Wallinga J, Lipsitch M: How generation intervals shape the relationship between growth rates and reproductive numbers. Proc Biol Sci 2007 274(1609):599-604.

15. Caley P, Philp DJ, McCracken K: Quantifying social distancing arising from pandemic influenza. Journal of the Royal Society, Interface / the Royal Society 2008, 5 (23):631-639.

16. Massad E, Burattini MN, Coutinho FA, Lopez LF: The 1918 influenza A epidemic in the city of Sao Paulo, Brazil. Med Hypotheses 2007, 68(2):442-445

17. Sattenspiel L: Regional patterns of mortality during the 1918 influenza pandemic in Newfoundland. Vaccine 2011, 29(Suppl 2):B33-B37.

18. Chowell G, Viboud C, Simonsen L, Miller MA, Acuna-Soto R, Diaz JM, Martinez-Martin AF: The 1918-19 influenza pandemic in Boyaca, Colombia. Emerg Infect Dis 2012, 18(1):48-56.

19. Andreasen V, Viboud C, Simonsen L: Epidemiologic characterization of the 1918 influenza pandemic summer wave in Copenhagen: implications for pandemic control strategies. J Infect Dis 2008, 197(2):270-278.

20. Rizzo C, Ajelli M, Merler S, Pugliese A, Barbetta I, Salmaso S, Manfredi P. Epidemiology and transmission dynamics of the 1918-19 pandemic influenza in Florence, Italy. Vaccine 2011, 29(Suppl 2):B27-B32.

21. Chowell G, Viboud C, Simonsen L, Miller MA, Acuna-Soto R: Mortality patterns associated with the 1918 influenza pandemic in Mexico: evidence for a spring herald wave and lack of preexisting immunity in older populations. J Infect Dis 2010, 202(4):567-575.

22. Chowell G, Viboud C, Simonsen L, Miller MA, Hurtado J, Soto G, Vargas R, Guzman MA, Ulloa M, Munayco CV: The 1918-1920 influenza pandemic in Peru. Vaccine 2011, 29 (Suppl 2):B21-B26.

23. Chowell G, Ammon CE, Hengartner NW, Hyman JM: Estimation of the reproductive number of the Spanish flu epidemic in Geneva, Switzerland. Vaccine 2006, 24(44-46):6747-6750

24. Rios-Doria D, Chowell G: Qualitative analysis of the level of cross-protection between epidemic waves of the 1918-1919 influenza pandemic. J Theor Biol 2009, 261(4):584-592.

25. Viboud C, Tam T, Fleming D, Handel A, Miller MA, Simonsen L: Transmissibility and mortality impact of epidemic and pandemic influenza, with emphasis on the unusually deadly 1951 epidemic Vaccine 2006, 24(44-46):6701-6707.

26. Gani R, Hughes H, Fleming D, Griffin T, Medlock J, Leach S: Potential impact of antiviral drug use during influenza pandemic. Emerg Infect Dis 2005, 11(9):1355-1362.

27. Mathews JD, McBryde ES, McVernon J, Pallaghy PK, McCaw JM: Prior immunity helps to explain wave-like behaviour of pandemic influenza in 1918-9. BMC Infect Dis 2010, 10:128.

28. White LF, Pagano M: Transmissibility of the influenza virus in the 1918 pandemic. PLoS One 2008, 3(1):e1498.

29. Vynnycky E, Trindall A, Mangtani P: Estimates of the reproduction numbers of Spanish influenza using morbidity data. Int J Epidemiol 2007, 36(4):881-889.

30. Zhang S, Yan P, Winchester B, Wang J: Transmissibility of the 1918 pandemic influenza in Montreal and Winnipeg of Canada. Influenza Other Respi Viruses 2010, 4(1):27-31.

31. Nishiura H: Time variations in the transmissibility of pandemic influenza in Prussia, Germany, from 1918-19. Theor Biol Med Model 2007, 4:20.

32. Sertsou G, Wilson N, Baker M, Nelson P, Roberts MG: Key transmission parameters of an institutional outbreak during the 1918 influenza pandemic estimated by mathematical modelling. Theor Biol Med Model 2006, 3:38

33. Chowell G, Bettencourt LM, Johnson N, Alonso WJ, Viboud C: The 1918-1919 influenza pandemic in England and Wales: spatial patterns in transmissibility and mortality impact. Proc Biol Sci 2008, 275(1634):501-509.

34. Goldstein E, Dushoff J, Ma J, Plotkin JB, Earn DJ, Lipsitch M: Reconstructing influenza incidence by deconvolution of daily mortality time series. Proc Natl Acad Sci U S A 2009, 106(51):21825-21829.

35. Chowell G, Nishiura H, Bettencourt LM: Comparative estimation of the reproduction number for pandemic influenza from daily case notification data. Journal of the Royal Society, Interface / the Royal Society 2007, 4(12):155-166.

36. Mills CE, Robins JM, Lipsitch M: Transmissibility of 1918 pandemic influenza. Nature 2004, 432(7019):904-906

37. Glezen WP: Emerging infections: pandemic influenza. Epidemiol Rev 1996, 18(1):64-76.

38. Nishiura $\mathrm{H}$ : Time variations in the generation time of an infectious disease: implications for sampling to appropriately quantify transmission potential. Mathematical biosciences and engineering : MBE 2010, 7(4):851-869.

39. Vynnycky E, Edmunds WJ: Analyses of the 1957 (Asian) influenza pandemic in the United Kingdom and the impact of school closures. Epidemiol Infect 2008, 136(2):166-179.

40. Longini IM Jr, Halloran ME, Nizam A, Yang Y: Containing pandemic influenza with antiviral agents. Am J Epidemiol 2004, 159(7):623-633.

41. Langmuir AD: Epidemiology of Asian influenza. With special emphasis on the United States. Am Rev Respir Dis 1961, 83(2):2-14. Pt 2.

42. Longini IM Jr, Fine PE, Thacker SB: Predicting the global spread of new infectious agents. Am J Epidemiol 1986, 123(3):383-391.

43. Jackson C, Vynnycky E, Mangtani P: Estimates of the transmissibility of the 1968 (Hong Kong) influenza pandemic: evidence of increased transmissibility between successive waves. Am J Epidemiol 2010, 171(4):465-478.

44. Hsieh YH, Ma S, Velasco Hernandez JX, Lee VJ, Lim WY: Early outbreak of 2009 influenza A (H1N1) in Mexico prior to identification of pH1N1 virus. PLoS One 2011, 6(8):e23853. 
45. Kelly HA, Mercer GN, Fielding JE, Dowse GK, Glass K, Carcione D, Grant KA, Effler PV, Lester RA: Pandemic (H1N1) 2009 influenza community transmission was established in one Australian state when the virus was first identified in North America. PLoS One 2010, 5(6):e11341.

46. McBryde E, Bergeri I, van Gemert C, Rotty J, Headley E, Simpson K, Lester R, Hellard M, Fielding J: Early transmission characteristics of influenza $A$ (H1N1)v in Australia: Victorian state, 16 May - 3 June 2009. Euro Surveill 2009, 14(42).

47. Buckley D, Bulger D: Estimation of the reproductive number for the 2009 pandemic H1N1 influenza in rural and metropolitan New South Wales. The Australian journal of rural health 2011, 19(2):59-63.

48. Hsieh YH, Fisman DN, Wu J: On epidemic modeling in real time: an application to the 2009 novel A (H1N1) influenza outbreak in Canada. BMC research notes 2010, 3:283.

49. Mostaco-Guidolin LC, Bowman CS, Greer AL, Fisman DN, Moghadas SM: Transmissibility of the: H1N1 pandemic in remote and isolated Canadian communities: a modelling study. BMJ open 2009, 2012:2(5).

50. Tuite AR, Greer AL, Whelan M, Winter AL, Lee B, Yan P, Wu J, Moghadas S, Buckeridge D, Pourbohloul B, Fisman DN: Estimated epidemiologic parameters and morbidity associated with pandemic H1N1 influenza. CMAJ 2010, 182(2):131-136.

51. Chilean Task Force for study of Pandemic Influenza A, Pedroni E, Garcia M, Espinola V, Guerrero A, Gonzalez C, Olea A, Calvo M, Martorell B, Winkler M, Carrasco M, Vergara J, Ulloa J, Carrazana A, Mujica O, Villarroel J, Labrana M, Vargas M, Gonzalez P, Caceres L, Zamorano C, Momberg R, Munoz G, Rocco J, Bosque V, Gallardo A, Elgueta J, Vega J: Outbreak of 2009 pandemic influenza A(H1N1), Los Lagos, Chile, April-June 2009. Euro Surveill 2010, 15(1).

52. Chowell G, Towers S, Viboud C, Fuentes R, Sotomayor V, Simonsen L, Miller MA, Lima M, Villarroel C, Chiu M, Villarroel JE, Olea A, Villarroel JE, Olea A: The influence of climatic conditions on the transmission dynamics of the 2009 A/H1N1 influenza pandemic in Chile. BMC Infect Dis 2012, 12:298.

53. Yu H, Cauchemez S, Donnelly CA, Zhou L, Feng L, Xiang N, Zheng J, Ye M, Huai Y, Liao Q, Peng Z, Feng Y, Jiang H, Yang W, Wang Y, Ferguson NM, Feng Z: Transmission dynamics, border entry screening, and school holidays during the 2009 influenza A (H1N1) pandemic. China Emerg Infect Dis 2012, 18(5):758-766.

54. Tan $X$, Yuan $L$, Zhou J, Zheng $Y$, Yang F: Modeling the initial transmission dynamics of influenza A H1N1 in Guangdong Province, China. International journal of infectious diseases: IIID : official publication of the International Society for Infectious Diseases 2012.

55. Jin Z, Zhang J, Song LP, Sun GQ, Kan J, Zhu H: Modelling and analysis of influenza A (H1N1) on networks. BMC Public Health 2011, 11(Suppl 1):S9.

56. Wu JT, Cowling BJ, Lau EH, Ip DK, Ho LM, Tsang T, Chuang SK, Leung PY, Lo SV, Liu SH, Riley S: School closure and mitigation of pandemic (H1N1) 2009, Hong Kong. Emerg Infect Dis 2010, 16(3):538-541.

57. Cowling BJ, Lau MS, Ho LM, Chuang SK, Tsang T, Liu SH, Leung PY, Lo SV, Lau EH: The effective reproduction number of pandemic influenza: prospective estimation. Epidemiology (Cambridge, Mass) 2010, 21(6):842-846

58. Katriel G, Yaari R, Huppert A, Roll U, Stone L: Modelling the initial phase of an epidemic using incidence and infection network data: 2009 H1N1 pandemic in Israel as a case study. Journal of the Royal Society, Interface / the Royal Society 2011, 8(59):856-867.

59. Dorigatti I, Cauchemez S, Pugliese A, Ferguson NM: A new approach to characterising infectious disease transmission dynamics from sentinel surveillance: application to the Italian 2009-2010 A/H1N1 influenza pandemic. Epidemics 2012, 4(1):9-21.

60. Nishiura H, Castillo-Chavez C, Safan M, Chowell G: Transmission potential of the new influenza $A(\mathrm{H} 1 \mathrm{~N} 1)$ virus and its age-specificity in Japan. Euro Surveill 2009, 14(22)

61. Fraser C, Donnelly CA, Cauchemez S, Hanage WP, Van Kerkhove MD, Hollingsworth TD, Griffin J, Baggaley RF, Jenkins HE, Lyons EJ, Jombart T, Hinsley WR, Grassly NC, Balloux F, Ghani AC, Ferguson NM, Rambaut A, Pybus OG, Lopez-Gatell H, Alpuche-Aranda CM, Chapela IB, Zavala EP, Guevara DM, Checchi F, Garcia E, Hugonnet S, Roth C, WHO Rapid Pandemic Assessment Collaboration: Pandemic potential of a strain of influenza A (H1N1): early findings. Science (New York, NY) 2009, 324(5934):1557-1561.

62. White LF, Pagano M: Reporting errors in infectious disease outbreaks, with an application to Pandemic Influenza A/H1N1. Epidemiologic perspectives \& innovations : $E P+1$ 2010, 7:12
63. Chowell G, Echevarria-Zuno S, Viboud C, Simonsen L, Tamerius J, Miller MA Borja-Aburto VH: Characterizing the epidemiology of the 2009 influenza A/H1N1 pandemic in Mexico. PLoS Med 2011, 8(5):e1000436.

64. Pourbohloul B, Ahued A, Davoudi B, Meza R, Meyers LA, Skowronski DM, Villasenor I, Galvan F, Cravioto P, Earn DJ, Dushoff J, Fisman D, Edmunds WJ, Hupert N, Scarpino SV, Trujillo J, Lutzow M, Morales J, Contreras A, Chávez C, Patrick DM, Brunham RC: Initial human transmission dynamics of the pandemic (H1N1) 2009 virus in North America. Influenza Other Respi Viruses 2009, 3(5):215-222.

65. Boelle PY, Bernillon P, Desenclos JC: A preliminary estimation of the reproduction ratio for new influenza $A(\mathrm{H} 1 \mathrm{~N} 1)$ from the outbreak in Mexico, March-April 2009. Euro Surveill 2009, 14(19).

66. Balcan D, Hu H, Goncalves B, Bajardi P, Poletto C, Ramasco JJ, Paolotti D, Perra N, Tizzoni M, Van den Broeck W, Colizza V, Vespignani A: Seasonal transmission potential and activity peaks of the new influenza $A(\mathrm{H} 1 \mathrm{~N} 1)$ : a Monte Carlo likelihood analysis based on human mobility. BMC medicine 2009, 7:45.

67. Cruz-Pacheco G, Duran L, Esteva L, Minzoni A, Lopez-Cervantes M, Panayotaros P, Ahued Ortega A, Villasenor Ruiz I: Modelling of the influenza A(H1N1)v outbreak in Mexico City, April-May 2009, with control sanitary measures. Euro Surveill 2009, 14(26).

68. Barakat A, Ihazmad H, El Falaki F, Tempia S, Cherkaoui I, El Aouad R: Pandemic influenza A virus subtype H1N1 in Morocco, 2009-2010: epidemiology, transmissibility, and factors associated with fatal cases. $J$ Infect Dis 2009, 2012(206 Suppl 1):S94-S100.

69. Hahne S, Donker T, Meijer A, Timen A, van Steenbergen J, Osterhaus A, van der Sande M, Koopmans M, Wallinga J, Coutinho R, Dutch New Influenza A (H1N1)v Investigation Team: Epidemiology and control of influenza A (H1N1)v in the Netherlands: the first 115 cases. Euro Surveill 2009, 14(27).

70. Roberts MG, Nishiura $\mathrm{H}$ : Early estimation of the reproduction number in the presence of imported cases: pandemic influenza H1N1-2009 in New Zealand. PLoS One 2011, 6(5):e17835.

71. Nishiura H, Wilson N, Baker MG: Estimating the reproduction number of the novel influenza A virus (H1N1) in a Southern Hemisphere setting: preliminary estimate in New Zealand. The New Zealand medical journal 2009, 122(1299):73-77.

72. Paine S, Mercer GN, Kelly PM, Bandaranayake D, Baker MG, Huang QS, Mackereth G, Bissielo A, Glass K, Hope V: Transmissibility of 2009 pandemic influenza $A(\mathrm{H} 1 \mathrm{~N} 1)$ in New Zealand: effective reproduction number and influence of age, ethnicity and importations. Euro Surveill 2010, 15(24).

73. Lessler J, dos Santos T, Aguilera X, Brookmeyer R, Group PITW, Cummings DA: H1N1pdm in the Americas. Epidemics 2010, 2(3):132-138.

74. Munayco CV, Gomez J, Laguna-Torres VA, Arrasco J, Kochel TJ, Fiestas V, Garcia J, Perez J, Torres I, Condori F, Nishiura H, Chowell G: and transmissibility analysis of influenza A(H1N1)v in a southern hemisphere setting: Peru Euro Sunveill 2009, 14(32).

75. Chowell G, Viboud C, Munayco CV, Gomez J, Simonsen L, Miller MA, Tamerius J, Fiestas V, Halsey ES, Laguna-Torres VA: Spatial and temporal characteristics of the $2009 \mathrm{~A} / \mathrm{H} 1 \mathrm{~N} 1$ influenza pandemic in Peru. PLoS One 2011, 6(6):e21287.

76. Chan PP, Subramony H, Lai FY, Tien WS, Tan BH, Solhan S, Han HK, Foong BH, James L, Ooi PL: Outbreak of novel influenza A (H1N1-2009) linked to a dance club. Ann Acad Med Singap 2010, 39(4):299-294.

77. Lee VJ, Yap J, Cook AR, Chen MI, Tay JK, Tan BH, Loh JP, Chew SW, Koh WH, Lin R, Cui L, Lee CW, Sung WK, Wong CW, Hibberd ML, Kang WL, Seet B, Tambyah PA: Oseltamivir ring prophylaxis for containment of 2009 H1N1 influenza outbreaks. N Engl J Med 2010, 362(23):2166-2174.

78. Archer BN, Tempia S, White LF, Pagano M, Cohen C: Reproductive number and serial interval of the first wave of influenza $A(\mathrm{H} 1 \mathrm{~N} 1)$ pdm09 virus in South Africa. PLoS One 2012, 7(11):e49482.

79. Hsieh YH: Pandemic influenza A (H1N1) during winter influenza season in the southern hemisphere. Influenza Other Respi Viruses 2010, 4(4):187-197.

80. Opatowski L, Fraser C, Griffin J, de Silva E, Van Kerkhove MD, Lyons EJ, Cauchemez S, Ferguson NM: Transmission characteristics of the 2009 H1N1 influenza pandemic: comparison of 8 Southern hemisphere countries. PLOS Pathog 2011, 7(9):e1002225.

81. Hsieh YH, Cheng KF, Wu TN, Li TC, Chen CY, Chen JH, Lin MH, Center for Infectious E, Research T: Transmissibility and temporal changes of 2009 $\mathrm{pH} 1 \mathrm{~N} 1$ pandemic during summer and fall/winter waves. BMC Infect Dis 2011, 11:332. 
82. Chao DY, Cheng KF, Li TC, Wu TN, Chen CY, Tsai CA, Chen JH, Chiu HT, Lu JJ, Su MC, Liao YH, Chan WC, Hsieh YH: Serological evidence of subclinical transmission of the 2009 pandemic H1N1 influenza virus outside of Mexico. PLoS One 2011, 6(1):e14555.

83. de Silva UC, Warachit J, Waicharoen S, Chittaganpitch M: A preliminary analysis of the epidemiology of influenza $A(H 1 N 1) v$ virus infection in Thailand from early outbreak data, June-July 2009. Euro Surveill 2009, 14(31).

84. Hens N, Calatayud L, Kurkela S, Tamme T, Wallinga J: Robust reconstruction and analysis of outbreak data: influenza $\mathrm{A}(\mathrm{H} 1 \mathrm{~N} 1) \mathrm{v}$ transmission in a school-based population. Am J Epidemiol 2012, 176(3):196-203.

85. Ghani A, Baguelin M, Griffin J, Flasche S, van Hoek AJ, Cauchemez S, Donnelly C, Robertson C, White M, Truscott J, Fraser C, Garske T, White P, Leach S, Hall I, Jenkins H, Ferguson N, Cooper B: The early transmission dynamics of H1N1pdm influenza in the United Kingdom. PLoS currents 2009, 1, RRN1130.

86. White LF, Wallinga J, Finelli L, Reed C, Riley S, Lipsitch M, Pagano M: Estimation of the reproductive number and the serial interval in early phase of the 2009 influenza A/H1N1 pandemic in the USA. Influenza Other Respi Viruses 2009, 3(6):267-276.

87. Lessler J, Reich NG, Cummings DA, New York City Department of H, Menta Hygiene Swine Influenza Investigation T, Nair HP, Jordan HT, Thompson N: Outbreak of 2009 pandemic influenza A (H1N1) at a New York City school. N Engl J Med 2009, 361(27):2628-2636.

88. Sugimoto JD, Borse NN, Ta ML, Stockman LJ, Fischer GE, Yang Y, Halloran ME, Longini IM Jr, Duchin JS: The effect of age on transmission of 2009 pandemic influenza A (H1N1) in a camp and associated households. Epidemiology (Cambridge, Mass) 2011, 22(2):180-187.

89. Hien TT, Boni MF, Bryant JE, Ngan TT, Wolbers M, Nguyen TD, Truong NT, Dung NT, Ha do Q, Hien VM, Thanh TT, le NT N, le TT U, Nhien PT, Chinh NT, Chau NV, Farrar J, van Doorn HR: Early pandemic influenza (2009 H1N1) in Ho Chi Minh City, Vietnam: a clinical virological and epidemiological analysis. PLoS Med 2010, 7(5):e1000277.

90. Hens N, Van Ranst M, Aerts M, Robesyn E, Van Damme P, Beutels P. Estimating the effective reproduction number for pandemic influenza from notification data made publicly available in real time: a multi-country analysis for influenza A/H1N1v 2009. Vaccine 2011, 29(5):896-904

91. Tang S, Xiao Y, Yuan L, Cheke RA, Wu J: Campus quarantine (Fengxiao) for curbing emergent infectious diseases: lessons from mitigating $A / H 1 N 1$ in Xi'an, China. J Theor Biol 2012, 295:47-58.

92. Tang S, Xiao Y, Yang Y, Zhou Y, Wu J, Ma Z: Community-based measures for mitigating the $2009 \mathrm{H} 1 \mathrm{~N} 1$ pandemic in China. PLoS One 2010, 5(6):e10911.

93. Trichereau J, Verret C, Mayet A, Manet G, Decam C, Meynard JB, Deparis X, Migliani R: Estimation of the reproductive number for $\mathrm{A}(\mathrm{H} 1 \mathrm{~N} 1)$ pdm09 influenza among the French armed forces, September 2009-March 2010. The Journal of infection 2012, 64(6):628-630.

94. Haghdoost A, Baneshi MR, Zolala F, Farvahari S, Safizadeh H: Estimation of basic reproductive number of Flu-like syndrome in a primary school in Iran. International journal of preventive medicine 2012 , 3(6):408-413.

95. Poletti $P$, Ajelli M, Merler S: The effect of risk perception on the 2009 H1N1 pandemic influenza dynamics. PLoS One 2011, 6(2):e16460.

96. Tsukui S: Case-Based Surveillance of Pandemic (H1N1) 2009 in Maebashi City, Japan. Jpn J Infect Dis 2012, 65(2):132-137.

97. Renault P, D'Ortenzio E, Kermarec F, Filleul L: Pandemic influenza 2009 on reunion island: a mild wave linked to a low reproduction number. PLoS currents 2010, 2, RRN1145.

98. Baguelin M, Hoek AJ, Jit M, Flasche S, White PJ, Edmunds WJ: Vaccination against pandemic influenza $\mathrm{A} / \mathrm{H} 1 \mathrm{~N} 1 \mathrm{v}$ in England: a real-time economic evaluation. Vaccine 2010, 28(12):2370-2384.

99. Glass K, Kelly H, Mercer GN: Pandemic influenza H1N1: reconciling serosurvey data with estimates of the reproduction number. Epidemiology (Cambridge, Mass) 2012, 23(1):86-94.

100. Van Kerkhove MD, Mounts AW, Mall S, Vandemaele KA, Chamberland M, dos Santos T, Fitzner J, Widdowson MA, Michalove J, Bresee J, et al: Epidemiologic and virologic assessment of the 2009 influenza A (H1N1) pandemic on selected temperate countries in the Southern Hemisphere: Argentina, Australia, Chile, New Zealand and South Africa. Influenza Other Respi Viruses 2011, 5(6):e487-e498.
101. Valleron AJ, Cori A, Valtat S, Meurisse S, Carrat F, Boelle PY: Transmissibility and geographic spread of the 1889 influenza pandemic. Proc Natl Acad Sci U S A 2010, 107(19):8778-8781.

102. Spicer CC: The mathematical modelling of influenza epidemics. Br Med Bull 1979, 35(1):23-28.

103. Chowell G, Miller MA, Viboud C: Seasonal influenza in the United States, France, and Australia: transmission and prospects for control. Epidemiol Infect 2008, 136(6):852-864.

104. Gran JM, Iversen B, Hungnes O, Aalen OO: Estimating influenza-related excess mortality and reproduction numbers for seasonal influenza in Norway, 1975-2004. Epidemiol Infect 2010, 138(11):1559-1568.

105. Britton T, Becker NG: Estimating the immunity coverage required to prevent epidemics in a community of households. Biostatistics (Oxford England) 2000, 1(4):389-402.

106. Wearing HJ, Rohani P, Keeling MJ: Appropriate models for the management of infectious diseases. PLoS Med 2005, 2(7):e174.

107. Flahault A, Letrait S, Blin P, Hazout S, Menares J, Valleron AJ: Modelling the 1985 influenza epidemic in France. Stat Med 1988, 7(11):1147-1155.

108. Truscott J, Fraser C, Hinsley W, Cauchemez S, Donnelly C, Ghani A, Ferguson N, Meeyai A: Quantifying the transmissibility of human influenza and its seasonal variation in temperate regions. PLoS currents 2009, 1, RRN1125.

109. Truscott J, Fraser C, Cauchemez S, Meeyai A, Hinsley W, Donnelly CA, Ghan A, Ferguson N: Essential epidemiological mechanisms underpinning the transmission dynamics of seasonal influenza. Journal of the Royal Society, Interface / the Royal Society 2012, 9(67):304-312.

110. Cauchemez S, Valleron AJ, Boelle PY, Flahault A, Ferguson NM: Estimating the impact of school closure on influenza transmission from Sentinel data. Nature 2008, 452(7188):750-754.

111. Chowell G, Viboud C, Simonsen L, Miller M, Alonso WJ: The reproduction number of seasonal influenza epidemics in Brazil, 1996-2006. Proc Biol Sci 2010, 277(1689):1857-1866.

112. Barnea $O$, Yaari R, Katriel G, Stone L: Modelling seasonal influenza in Israel. Mathematical biosciences and engineering: MBE 2011, 8(2):561-573.

113. Cintron-Arias A, Castillo-Chavez C, Bettencourt LM, Lloyd AL, Banks HT: The estimation of the effective reproductive number from disease outbreak data. Mathematical biosciences and engineering: MBE 2009, 6(2):261-282.

114. Huppert A, Barnea O, Katriel G, Yaari R, Roll U, Stone L: Modeling and statistical analysis of the spatio-temporal patterns of seasonal influenza in Israel. PLoS One 2012, 7(10):e45107.

115. Chen SC, Liao CM: Probabilistic indoor transmission modeling for influenza (sub)type viruses. The Journal of infection 2010, 60(1):26-35.

116. Lunelli A, Rizzo C, Puzelli S, Bella A, Montomoli E, Rota MC, Donatelli I, Pugliese A: Understanding the dynamics of seasonal influenza in Italy: incidence, transmissibility and population susceptibility in a 9-year period. Influenza Other Respi Viruses 2013, 7(2):286-295.

117. Smieszek T, Balmer M, Hattendorf J, Axhausen KW, Zinsstag J, Scholz RW: Reconstructing the 2003/2004 H3N2 influenza epidemic in Switzerland with a spatially explicit, individual-based model. BMC Infect Dis 2011 $11: 115$.

118. Hsieh YH: Age groups and spread of influenza: implications for vaccination strategy. BMC Infect Dis 2010, 10:106.

119. Bettencourt LM, Ribeiro RM: Real time bayesian estimation of the epidemic potential of emerging infectious diseases. PLOS One 2008, 3(5):e2185.

120. Borja-Aburto VH, Chowell G, Viboud C, Simonsen L, Miller MA, GrajalesMuniz C, Gonzalez-Bonilla CR, Diaz-Quinonez JA, Echevarria-Zuno S: Epidemiological characterization of a fourth wave of pandemic A/H1N1 influenza in Mexico, winter 2011-2012: age shift and severity. Arch Med Res 2012, 43(7):563-570.

121. Chowell G, Echevarria-Zuno S, Viboud C, Simonsen L, Grajales Muniz C, Rascon Pacheco RA, Gonzalez Leon M, Borja Aburto VH: Recrudescent wave of pandemic A/H1N1 influenza in Mexico, winter 2011-2012: Age shift and severity. PLOS currents 2012, 4, RRN1306.

122. Lessler J, Cummings DA, Fishman S, Vora A, Burke DS: Transmissibility of swine flu at Fort Dix, 1976. Journal of the Royal Society, Interface / the Royal Society 2007, 4(15):755-762.

123. Yang $Y$, Halloran ME, Sugimoto JD, Longini IM Jr: Detecting human-tohuman transmission of avian influenza A (H5N1). Emerg Infect Dis 2007, 13(9):1348-1353.

124. Aditama TY, Samaan G, Kusriastuti R, Sampurno OD, Purba W, Misriyah, Santoso H, Bratasena A, Maruf A, Sariwati E, Setiawaty V, Glass K, Lokuge K, 
Kelly PM, Kandun IN: Avian influenza H5N1 transmission in households, Indonesia. PLoS One 2012, 7(1):e29971.

125. Frost WH: The epidemiology of influenza. J Am Med Assoc 1919, 73(5):313-318

126. Shrestha SS, Swerdlow DL, Borse RH, Prabhu VS, Finelli L, Atkins CY, Owusu-Edusei K, Bell B, Mead PS, Biggerstaff M, Brammer L, Davidson H, Jernigan D, Jhung MA, Kamimoto LA, Merlin TL, Nowell M, Redd SC, Reed C, Schuchat A, Meltzer MI: Estimating the burden of 2009 pandemic influenza A (H1N1) in the United States (April 2009-April 2010). Clin Infect Dis 2011, 52(Suppl 1):S75-S82.

127. Cumulative number of confirmed human cases of avian influenza $\mathrm{A}(\mathrm{H} 5 \mathrm{~N} 1)$ reported to WHO. [http://www.who.int/influenza/human_animal_interface/ EN_GIP_20130215CumulativeNumberH5N1cases.pdf]

128. Mossong J, Hens N, Jit M, Beutels P, Auranen K, Mikolajczyk R, Massari M, Salmaso S, Tomba GS, Wallinga J, Heijne J, Sadkowska-Todys M, Rosinska M, Edmunds WJ: Social contacts and mixing patterns relevant to the spread of infectious diseases. PLoS Med 2008, 5(3):e74.

129. The world factbook. [https://www.cia.gov/library/publications/the-worldfactbook/]

130. Nishiura H, Chowell G, Castillo-Chavez C: Did modeling overestimate the transmission potential of pandemic (H1N1-2009)? Sample size estimation for post-epidemic seroepidemiological studies. PLoS One 2011, 6(3):e17908.

131. Shaman J, Kohn M: Absolute humidity modulates influenza survival, transmission, and seasonality. Proc Natl Acad Sci U S A 2009, 106(9):3243-3248.

132. Shaman J, Pitzer V, Viboud C, Lipsitch M, Grenfell B: Absolute humidity and the seasonal onset of influenza in the continental US. PLoS currents 2009, 2, RRN1138.

133. Influenza in a boarding school: News and Notes. BMJ 1978, 1:587.

doi:10.1186/1471-2334-14-480

Cite this article as: Biggerstaff et al:: Estimates of the reproduction number for seasonal, pandemic, and zoonotic influenza: a systematic review of the literature. BMC Infectious Diseases 2014 14:480.

\section{Submit your next manuscript to BioMed Central and take full advantage of:}

- Convenient online submission

- Thorough peer review

- No space constraints or color figure charges

- Immediate publication on acceptance

- Inclusion in PubMed, CAS, Scopus and Google Scholar

- Research which is freely available for redistribution 